\title{
Dark neutrinos and a three-portal connection to the standard model
}

\author{
Peter Ballett, ${ }^{1}$ Matheus Hostert $\odot,{ }^{2,3,4, *}$ and Silvia Pascoli ${ }^{1, \dagger}$ \\ ${ }^{1}$ Institute for Particle Physics Phenomenology, Department of Physics, Durham University, \\ South Road, Durham DH1 3LE, United Kingdom \\ ${ }^{2}$ School of Physics and Astronomy, University of Minnesota, Minneapolis, Minnesota 55455, USA \\ ${ }^{3}$ William I. Fine Theoretical Physics Institute, School of Physics and Astronomy, \\ University of Minnesota, Minneapolis, Minnesota 55455, USA \\ ${ }^{4}$ Perimeter Institute for Theoretical Physics, Waterloo, Ontario N2J 2W9, Canada
}

(Received 27 March 2019; revised manuscript received 24 March 2020; accepted 2 June 2020; published 19 June 2020)

\begin{abstract}
We introduce a dark neutrino sector which respects a hidden $U(1)^{\prime}$ gauge symmetry, subsequently broken by the vacuum expectation value of a dark scalar. The model is a self-consistent realization of an extended hidden sector that communicates with the standard model only via the three renormalizable portals, namely, neutrino, vector and scalar mixing. The interplay between portal couplings leads to several novel signatures in heavy neutrino, dark photon, and dark scalar searches, typically characterized by multileptons plus missing energy and displaced vertices. A striking signature arises in kaon factories such as NA62, where $K^{+} \rightarrow \ell_{\alpha}^{+} \nu \ell_{\beta}^{+} \ell_{\beta}^{-}$decays could reveal a heavy neutrino and a light dark photon resonance above backgrounds. Given the open parameter space, we also comment on recent ideas to explain outstanding experimental anomalies, and how they would fit into our proposed model. A minimal extension of the model, possibly motivated by anomaly cancellation, can accommodate a dark matter candidate strongly connected to the neutrino sector.
\end{abstract}

DOI: 10.1103/PhysRevD.101.115025

\section{INTRODUCTION}

The most important evidence that the standard model (SM) of particle physics is incomplete comprises neutrino masses and mixing, and the presence of dark matter (DM) in the Universe. Both call for extensions of the SM and the possible existence of dark sectors which do not partake in SM interactions, or do so with extremely weak couplings while displaying strong "dark" interactions [1-3]. Such sectors might exist at relatively light scales below the electroweak one, being within reach of present and future noncollider experiments. Generically, a neutral dark sector can communicate with the SM via three renormalizable portals. New neutral fermions mix with light neutrinos unless a symmetry differentiates the two, a possibility usually denoted as the neutrino portal. New vector particles can kinetically mix with the SM hypercharge, and new scalars mix with the Higgs boson through the so-called vector and scalar portals, respectively. The latter terms are

\footnotetext{
*mhostert@umn.edu

‘silvia.pascoli@durham.ac.uk
}

Published by the American Physical Society under the terms of the Creative Commons Attribution 4.0 International license. Further distribution of this work must maintain attribution to the author(s) and the published article's title, journal citation, and DOI. Funded by SCOAP. generically allowed in the Lagrangian and an explanation of their smallness requires specific UV completions.

In this article, we propose a new neutrino model with a hidden $U(1)^{\prime}$ gauge symmetry under which no SM fields are charged. We introduce new SM-neutral fermions $\nu_{D}$ and an additional sterile neutrino $N$. The symmetry is subsequently broken by the vacuum expectation value (VEV) of a complex dark scalar $\Phi$, which gives mass to the new gauge boson. For concreteness, we restrict the scale of the breaking to be below the electroweak one.

Models with heavy neutrinos which are not completely sterile and might participate in new gauge interactions have been studied in several contexts, including $B-L, L_{\mu}-L_{\tau}$, and left-right symmetric models [4-12], but here we focus on the possibility of a symmetry under which no SM fields are charged [13-17]. New heavy neutral fermions that feel such hidden forces, such as $\nu_{D}$, are referred to as dark neutrinos, since they define a dark sector separate from the SM. Nevertheless, the dark interactions "leak" into the SM sector via neutrino mixing, where they may dominate $[18,19]$. Models of this type have been invoked to generate large neutrino nonstandard interactions [20,21], generate new signals in DM experiments [18,22-25], weaken cosmological and terrestrial bounds on $\mathrm{eV}$-scale sterile neutrinos [26-33], and as a potential explanation of anomalous short-baseline results at the MiniBooNE [34,35] and/or LSND [36,37] 
experiments with new degrees of freedom at the $\mathrm{MeV} / \mathrm{GeV}$ scale [38-44].

Our model presents all the three renormalizable portals to the SM in a self-consistent way (see Fig. 1). The Yukawa interactions between the leptonic doublet and $N$, and between $N$ and $\nu_{D}$ induce neutrino mixing. The gauge symmetry allows a cross-coupling term in the potential between the Higgs and the real part of the scalar, inducing mixing between the two after symmetry breaking. The broken gauge symmetry implies the existence of a light hidden gauge boson $X_{\mu}$, which mediates the dark neutrino interactions and generically kinetically mixes with the SM hypercharge. This self-consistent setup combines the three portals into a unified picture that exhibits significantly different phenomenology with respect to each portal taken separately. Of particular interest is the fact that the different portal degrees of freedom display novel decay channels and scattering properties, and in many cases would have escaped experimental searches performed to date. We provide a selective list of the most affected bounds, and highlight the most interesting novel signatures that arise. In particular, we propose a new experimental search for the simultaneous presence of heavy neutrino and vector resonances in rare leptonic meson decays. In view of the relatively unexplored parameter space of our model, we comment on how these particles can explain long-standing experimental anomalies. We focus on a novel explanation of the MiniBooNE anomaly, based on the discussion of Ref. [42] (see also Refs. [43,44]), where new neutrino scattering signatures arise at neutrino experiments. We also reconsider the possibility to explain the discrepancy between the prediction [45-49] and measurement [50] of the anomalous magnetic moment of the muon $\left(\Delta a_{\mu}\right)$ via kinetic mixing [51,52]. Finally, we comment on how a scalar that couples strongly to dark neutrinos can help explain the anomalous $K_{L} \rightarrow \pi^{0} \nu \bar{\nu}$ events at KOTO [53].

An interesting feature of the model is the generation of neutrino masses at loop level. This requires only two key features of our setup, namely, a light $Z^{\prime}$ and neutrino mixing, but not the vector and scalar portals. For this reason, we discuss it elsewhere [54].

In its minimal form, the model is not anomaly free. We discuss how this can be cured and propose a minor extension that introduces additional dark sector neutral fermions charged under the new symmetry [1,2]. Neutrinos, we argue, may be a window into such dark sectors, bridging the puzzles of neutrino masses and DM [55-65]. We briefly outline the key features of a DM extension and leave a more detailed analysis to future work.

\section{THE MODEL}

We extend the SM gauge group with a new Abelian gauge symmetry $U(1)^{\prime}$ with associated mediator $X_{\mu}$ and introduce three new singlets of the SM gauge group: a complex scalar $\Phi$, and two left-handed fermions $\nu_{D, L} \equiv \nu_{D}$ and $N_{L} \equiv N$. The scalar $\Phi$ and the fermion $\nu_{D}$ are equally charged under the new symmetry, and $N$ is neutral with respect to all gauge symmetries of the model. For simplicity, we restrict our discussion to a single generation of hidden fermions. The relevant terms in the gauge-invariant Lagrangian are

$$
\begin{aligned}
\mathcal{L} \supset & \left(D_{\mu} \Phi\right)^{\dagger}\left(D^{\mu} \Phi\right)-V(\Phi, H) \\
& -\frac{1}{4} X^{\mu \nu} X_{\mu \nu}+\bar{N} i \not \partial N+\overline{\nu_{D}} i \not D \nu_{D} \\
& -\left[y_{\nu}^{\alpha}\left(\overline{L_{\alpha}} \cdot \tilde{H}\right) N^{c}+\frac{\mu^{\prime}}{2} \bar{N} N^{c}+y_{N} \bar{N} \nu_{D}^{c} \Phi+\text { H.c. }\right],
\end{aligned}
$$

where $X^{\mu \nu}$ is the field-strength tensor for $X_{\mu}, D_{\mu} \equiv$ $\left(\partial_{\mu}-i g^{\prime} X_{\mu}\right)$ is the covariant derivative, $L_{\alpha} \equiv\left(\nu_{\alpha}^{T}, \ell_{\alpha}^{T}\right)^{T}$ is the SM leptonic doublet of flavor $\alpha=e, \mu, \tau$, and $\tilde{H} \equiv$ $i \sigma_{2} H^{*}$ is the charge conjugate of the SM Higgs doublet. We write $y_{\nu}^{\alpha}$ for the $L_{\alpha}-N$ Yukawa coupling, $y_{N}$ for the $\nu_{D}-N$ one, and $\mu^{\prime}$ for the Majorana mass of $N$, which is allowed by the SM and the new gauge interaction, although it breaks lepton number by 2 units.

The minimization of the scalar potential $V(\Phi, H)$ causes the neutral component of the fields $H$ and $\Phi$ to acquire VEVs $v_{H}$ and $v_{\varphi}$, respectively. The latter also generates a mass for both the new gauge boson $X_{\mu}$ and the real component of the scalar field $\varphi$. Although $v_{\varphi}$ is arbitrary, we choose it to be below the electroweak scale, $v_{\varphi}<v_{H}$, as we are interested in building a model that is testable at low scales.

\section{A. Neutrino portal}

In the neutral fermion sector and after symmetry breaking, two Dirac mass terms are induced with $m_{D} \equiv$ $y_{\nu}^{\alpha} v_{H} / \sqrt{2}$ and $\Lambda \equiv y_{N} v_{\varphi} / \sqrt{2}$. It is useful to consider the form of the neutrino mass matrix in the single-generation case to clarify its main features. For one active neutrino $\nu_{\alpha}$ $(\alpha=e, \mu, \tau)$, it reads

$\mathcal{L}_{\text {mass }} \supset \frac{1}{2}\left(\begin{array}{lll}\bar{\nu}_{\alpha} & \bar{N} & \overline{\nu_{D}}\end{array}\right)\left(\begin{array}{ccc}0 & m_{D} & 0 \\ m_{D} & \mu^{\prime} & \Lambda \\ 0 & \Lambda & 0\end{array}\right)\left(\begin{array}{c}\nu_{\alpha}^{c} \\ N^{c} \\ \nu_{D}^{c}\end{array}\right)+$ H.c.

The form of this matrix appears in inverse seesaw (ISS) $[66,67]$ and in extended seesaw (ESS) $[68,69]$ models. In fact, it is the same matrix discussed in the so-called minimal ISS [70], with the difference that in our case its structure is a consequence of the hidden symmetry. After diagonalization of the mass matrix, the two heavy neutrinos $\nu_{h}$ (with $h=4,5)$ acquire masses. Assuming that $m_{D} \ll \Lambda$, we focus on two interesting limiting cases. 
In the ISS-like limit, where $\Lambda \gg \mu^{\prime}$ and the two heavy neutrinos are nearly degenerate, we have

$$
\begin{gathered}
m_{5} \simeq-m_{4} \simeq \Lambda, \quad m_{5}-\left|m_{4}\right|=\mu^{\prime}, \quad U_{\alpha 5} \simeq U_{\alpha 4} \simeq \frac{m_{D}}{\sqrt{2} \Lambda}, \\
U_{D i} \simeq \frac{m_{D}}{\Lambda}, \quad U_{D 5} \simeq U_{D 4} \simeq \frac{1}{\sqrt{2}}, \quad U_{N 5} \simeq U_{N 4} \simeq \frac{1}{\sqrt{2}} .
\end{gathered}
$$

In the ESS-like case, $\Lambda \ll \mu^{\prime}$, one heavy neutrino remains very heavy and mainly in the completely neutral direction $N$, and the other acquires a small mass via the seesaw mechanism in the hidden sector. We find

$$
\begin{aligned}
& m_{4} \simeq-\frac{\Lambda^{2}}{\mu^{\prime}}, \quad m_{5} \simeq \mu^{\prime}, \quad U_{\alpha 4} \simeq U_{\alpha 5} \sqrt{\frac{m_{5}}{\left|m_{4}\right|}} \simeq \frac{m_{D}}{\Lambda}, \\
& U_{D i} \simeq \frac{m_{D}}{\Lambda}, \quad U_{N 5} \simeq U_{D 4} \simeq 1, \quad U_{D 5} \simeq U_{N 4} \simeq \frac{\Lambda}{\mu^{\prime}} .
\end{aligned}
$$

Note that to lower the scale of the mediators while keeping the heavy neutrino masses large, one must lower the gauge and scalar quartic coupling. Finally, we note that the mixing parameters of $\nu_{4}$ and $\nu_{5}$ are correlated in our model. At tree level, the relation

$$
\frac{\left|U_{a 5}\right|^{2}}{\left|U_{a 4}\right|^{2}}=\frac{m_{4}}{m_{5}}
$$

holds exactly for $a=\alpha, N, D$. Loop corrections are expected to lead to small deviations from this relation. In practice, this implies that $\nu_{4}$ has typically larger activeheavy mixing than $\nu_{5}$.

The Yukawa terms in Eq. (1) induce neutrino mixing between the active (light) and heavy (sterile, dark) neutrinos. In this model, similarly to the ISS and ESS cases, this mixing can be much larger than the typical values required in type-I seesaw extensions to explain neutrino masses, making its phenomenology more interesting. The determinant of the mass matrix in Eq. (2) is zero, and so light neutrino masses vanish at tree level and do not constrain the values of the active-heavy mixing angles. This, however, is no longer the case at the one-loop level, as light neutrino masses emerge through radiative corrections from diagrams involving the $\varphi^{\prime}$ and $Z^{\prime}$ particles, as well as SM bosons [54].

\section{B. Scalar portal}

In the scalar potential, the symmetries of the model allow us to write down the following term:

$$
V(\Phi, H) \supset \lambda_{\Phi H} H^{\dagger} H|\Phi|^{2},
$$

where we identify $\lambda_{\Phi H}$ as the scalar portal coupling [71], responsible for mixing in the neutral scalar sector. If such a term exists, the scalar mass eigenstates $\left(h^{\prime}, \varphi^{\prime}\right)$ mix with the gauge eigenstates $(h, \varphi)$ as $h^{\prime}=h \cos \theta-\varphi \sin \theta$ and $\varphi^{\prime}=h \sin \theta+\varphi \cos \theta$, with a mixing angle $\theta$ defined by

$$
\tan (2 \theta) \equiv \frac{\lambda_{\Phi H} v_{H} v_{\varphi}}{\lambda_{H} v_{H}^{2}-\lambda_{\varphi} v_{\varphi}^{2}},
$$

where $\lambda_{H}$ and $\lambda_{\varphi}$ are the quartic couplings of the Higgs and $\Phi$ scalars, respectively.

\section{Vector portal}

Similarly, mixing also arises in the neutral vector boson sector from the allowed kinetic mixing term [72]

$$
\mathcal{L} \supset-\frac{\sin \chi}{2} F^{\mu \nu} X_{\mu \nu}
$$

where $F_{\mu \nu}$ is the SM hypercharge field strength. This term may be removed with a field redefinition, resulting in three mass eigenstates $\left(A, Z^{0}, Z^{\prime}\right)$, corresponding to the photon, $Z^{0}$ boson, and the hypothetical $Z^{\prime}$ boson. For a light $Z^{\prime}$, the $Z^{\prime}$ coupling to SM fermions $f$ to first order in the small parameter $\chi$ is given by

$$
\mathcal{L} \supset-\left(e q_{f} c_{W}\right) \chi \bar{f} \gamma^{\mu} f Z_{\mu}^{\prime},
$$

where $q_{f}$ is the fermion electric charge.

The values of $\chi$ and $\lambda_{\Phi H}$ are arbitrary and could be expected to be rather large. As such, we treat them as free parameters within their allowed ranges. Here, we merely note that with our current minimal matter content, $\chi$ and $\lambda_{\Phi H}$ receive contributions at loop level from the $\left(\bar{L}_{\alpha} \cdot \tilde{H}\right) N^{c}$ and $\bar{N} \nu_{D}^{c} \Phi$ terms, which are necessarily suppressed by neutrino mixing $\left(\chi \propto g^{\prime} e\left|U_{\alpha h}\right|^{2}\right.$ and $\left.\lambda_{\Phi H} \propto\left|U_{\alpha h}\right|^{2}\right)$. These values constitute a lower bound and larger values should be expected in a complete model.

\section{DECAY RATES}

The phenomenology of the model depends critically on the ordering of the heavy neutrinos and the dark bosons, which controls the decay channels and lifetimes of these particles. In what follows, we list the most relevant decay rates in our model, denoting by $\nu$ the combination of all light mass eigenstates $\left(\nu_{1}, \nu_{2}, \nu_{3}\right)$ that can appear in a given process. For clarity and simplicity, we separate the light dark boson and heavy dark boson cases, defined by the condition $m_{Z^{\prime}, \varphi^{\prime}}<m_{4}$ or $m_{4}<m_{Z^{\prime}, \varphi^{\prime}}$, respectively. We ignore SM contributions to the decay rates, as these are typically subleading for the cases of interest.

\section{A. Heavy bosons}

For $Z^{\prime}$ heavier than the heavy neutrinos, $m_{\nu_{h}}<m_{Z^{\prime}}$ with $h=4,5$, and with large kinetic mixing, the heavy neutrinos decay predominantly via three-body decays with 
an off-shell boson. Unless specified, we assume that the mass of the dark scalar is heavier and does not contribute to the decay rates in this section. The SM $Z$ and $Z^{\prime}$ contributions can interfere, although we are mainly interested in the case where the latter dominates. The decays of most interest are $\nu_{5} \rightarrow \nu_{4} \ell^{+} \ell^{-}$and $\nu_{4} \rightarrow \nu \ell^{+} \ell^{-}$, with $\ell=e, \mu$, as far as these channels are kinematically accessible. The decay length of $\nu_{5}$ critically depends on the mass difference between $\nu_{5}$ and $\nu_{4}$. For concreteness, we focus on specific benchmark points (BPs) that illustrate the key features. In the ISS-like regime, we take $m_{4} / m_{5}=$ $99 \%$ and choose $m_{4} \simeq m_{5}=100 \mathrm{MeV}$. If $\chi$ is negligible, we have that $\nu_{h}$ decays as in the minimal sterile neutrino model case via SM interactions. This is because the $\nu_{5} \rightarrow$ $\nu_{4} \nu \bar{\nu}$ decay is phase-space suppressed $\left(\Gamma_{\nu_{5} \rightarrow \nu_{4} \nu \nu} \propto \mu^{\prime 5}\right)$, and because $Z^{\prime}$-mediated decays into three light neutrinos are negligible for small mixing, as $\Gamma_{\nu_{h} \rightarrow \nu \nu \nu} \propto\left|U_{\alpha h}\right|^{6} m_{\nu_{h}}^{5} / m_{Z^{\prime}}^{4}$, where $\left|U_{\alpha h}\right|$ is a small mixing parameter between $e, \mu$, and $\tau$ flavors with the heavy neutrinos. If $\chi$ is sizable, on the other hand, new visible decay channels dominate, specifically $\nu_{5} \rightarrow \nu e^{+} e^{-}$and $\nu_{4} \rightarrow \nu e^{+} e^{-}$for this BP. On the other hand, in an ESS-like regime where $m_{4}=m_{5} / 10$, for instance, $\nu_{5}$ decays into three $\nu_{4}$ states very rapidly. The subsequent decays of $\nu_{4}$ would proceed via neutrino and kinetic mixing, but would be much slower than the $\nu_{5}$ one given the hierarchy of masses and the further suppression due to the portal couplings.

The most relevant decay rate for our case studies is that of heavy neutrinos into a dilepton plus missing energy. Neglecting the electron mass, the decay rate of $\nu_{5}$ is given by

$$
\begin{aligned}
\Gamma\left(\nu_{5} \rightarrow \nu_{4} e^{+} e^{-}\right) \simeq & \left|U_{D 5}\right|^{2}\left|U_{D 4}\right|^{2} F\left(\sqrt{x_{4}}\right) \\
& \times \frac{\left(e c_{W} \chi g^{\prime}\right)^{2}}{384 \pi^{3}} \frac{m_{5}^{5}}{m_{Z^{\prime}}^{4}},
\end{aligned}
$$

where $x_{4}=m_{4}^{2} / m_{5}^{2}$ and $F(x)=1+2 x-8 x^{2}+18 x^{3}-$ $18 x^{5}+8 x^{6}-2 x^{7}-x^{8}+24 x^{3}\left(1-x+x^{2}\right) \log x$. Note that $F(0)=1$ and $F(1)=0$, with the function obtaining a maximum value of $F(0.09) \simeq 1.09$. For $\sqrt{x_{4}}=1-\epsilon$, one can show that $F\left(x_{4}\right) \rightarrow \epsilon^{5}$ when $\epsilon \ll 1$, indicating a strong suppression of this decay in ISS-like scenarios, as stated before. Both $\nu_{4}$ and $\nu_{5}$ may decay via

$$
\begin{aligned}
\Gamma\left(\nu_{h} \rightarrow \nu e^{+} e^{-}\right) \simeq & \left|U_{D h}\right|^{2}\left(1-\left|U_{D 4}\right|^{2}-\left|U_{D 5}\right|^{2}\right) \\
& \times \frac{\left(e c_{W} \chi g^{\prime}\right)^{2}}{384 \pi^{3}} \frac{m_{h}^{5}}{m_{Z^{\prime}}^{4}} .
\end{aligned}
$$

For heavier masses, additional decay channels, e.g., $\nu_{4} \rightarrow \nu \mu^{+} \mu^{-}$and $\nu_{4} \rightarrow \nu \pi^{+} \pi^{-}$, would become available. A feature of the model is that $\nu_{4} \rightarrow \nu \mu^{+} \mu^{-}$would have the same branching ratio (BR) as the $e^{+} e^{-}$one, albeit phase space suppressed. Two-body decays into neutral pseudoscalars via the new force are heavily suppressed due to the vector nature of the gauge coupling, unless mass mixing with the $Z$ is introduced (see Ref. [73] for a thorough discussion of the decay products of a dark photon). Decays into vector mesons are enhanced, where, for instance, we find

$$
\begin{aligned}
\Gamma\left(\nu_{h} \rightarrow \nu \rho^{0}\right)= & \left|U_{D h}\right|^{2}\left(1-\left|U_{D 4}\right|^{2}-\left|U_{D 5}\right|^{2}\right) \\
& \times \frac{\left(e c_{W} \chi g^{\prime}\right)^{2}}{16 \pi} \frac{m_{h}^{3} f_{\rho}^{2}}{m_{Z^{\prime}}^{4}}\left(1-r_{\rho}\right)^{2}\left(\frac{1}{2}+r_{\rho}\right)
\end{aligned}
$$

for sufficiently heavy dark photons, and where $r_{\rho}=$ $m_{\rho}^{2} / m_{h}^{2}$ and $f_{\rho} \simeq 210 \mathrm{MeV}$.

The dominant decays of the heavy dark photon and dark scalar are into heavy neutrinos, and the relevant rates can be obtained from

$$
\begin{aligned}
\Gamma\left(Z^{\prime} \rightarrow \nu_{4} \nu_{5}\right)= & \left|U_{D 5}\right|^{2}\left|U_{D 4}\right|^{2} \frac{g^{\prime 2} m_{Z^{\prime}}}{12 \pi} \\
& \times\left(1+\frac{\Delta r}{2}\right)(1-R)^{3 / 2} \sqrt{1-\Delta r},
\end{aligned}
$$

where $R=\left(m_{4}+m_{5}\right)^{2} / m_{Z^{\prime}}^{2}$ and $\Delta r=\left(m_{5}-m_{4}\right)^{2} / m_{Z^{\prime}}^{2}$, and

$$
\begin{aligned}
\Gamma\left(\varphi^{\prime} \rightarrow \nu_{4} \nu_{5}\right)= & \left|U_{D 5} U_{N 4}+U_{D 4} U_{N 5}\right|^{2} \frac{y_{N}^{2} m_{\varphi^{\prime}}}{16 \pi} \\
& \times\left(1-R^{\prime}\right)^{3 / 2} \sqrt{1-\Delta r^{\prime}},
\end{aligned}
$$

where $R^{\prime}=\left(m_{4}+m_{5}\right)^{2} / m_{\varphi^{\prime}}^{2}$ and $\Delta r^{\prime}=\left(m_{5}-m_{4}\right)^{2} / m_{\varphi^{\prime}}^{2}$. Note that as a consequence of $U_{N i}=0$ for $i=1,2,3$, the decay $\varphi^{\prime} \rightarrow \nu \bar{\nu}$ vanishes at tree level, and can be neglected here.

\section{B. Light bosons}

If $m_{Z^{\prime}}<m_{\nu_{h}}$, the heavy neutrino will dominantly and immediately decay as $\nu_{h} \rightarrow \nu_{\alpha} Z^{\prime}$, or even faster for $\nu_{5} \rightarrow$ $\nu_{4} Z^{\prime}$ if this is kinematically allowed. For instance, the former decay rate is given by

$$
\begin{aligned}
\Gamma\left(\nu_{h} \rightarrow \nu Z^{\prime}\right)= & \left|U_{D h}\right|^{2}\left(1-\left|U_{D 4}\right|^{2}-\left|U_{D 5}\right|^{2}\right) \\
& \times \frac{g^{\prime 2}}{8 \pi} \frac{m_{\nu_{h}}^{3}}{m_{Z^{\prime}}^{2}}(1-r)^{2}\left(\frac{1}{2}+r\right),
\end{aligned}
$$

where $r=m_{Z^{\prime}}^{2} / m_{\nu_{h}}^{2}$, which agrees with the rate in Ref. [74] under the substitutions $Z^{\prime} \rightarrow Z$ and $g^{\prime} \rightarrow g / 2 c_{W}$, but differs from the rate in Ref [43]. For (pseudo-)Dirac $\nu_{h}$, the rate is smaller by a factor of 2 . If the scalar is light, $m_{\varphi^{\prime}}<m_{\nu_{h}}$, decay into the scalar degree of freedom is also possible, with 


$$
\begin{aligned}
\Gamma\left(\nu_{h} \rightarrow \nu \varphi^{\prime}\right)= & \left|U_{N h}\right|^{2}\left(1-\left|U_{D 4}\right|^{2}-\left|U_{D 5}\right|^{2}\right) \\
& \times \frac{y_{N}^{2}}{16 \pi} \frac{m_{\nu_{h}}^{3}}{m_{\phi^{\prime}}^{2}}(1-r)^{2}\left(\frac{1}{2}+r\right),
\end{aligned}
$$

where $r=m_{Z^{\prime}}^{2} / m_{\nu_{h}}^{2}$. The $Z^{\prime}$ subsequently decays into $e^{+} e^{-}$ via kinetic mixing with a decay rate

$\Gamma\left(Z^{\prime} \rightarrow \ell^{+} \ell^{-}\right)=\frac{\left(e c_{W} \chi\right)^{2}}{12 \pi} m_{Z^{\prime}}\left(1+2 r_{\ell}\right) \sqrt{1-4 r_{\ell}}$,

where $r_{\ell}=m_{\ell}^{2} / m_{Z^{\prime}}^{2}$. An analogous expression holds for $\varphi^{\prime} \rightarrow \ell^{+} \ell^{-}$decays, which are long-lived due to the $m_{\ell}^{2} / v_{H}^{2}$ suppression from the Higgs Yukawa coupling. It is given by

$$
\Gamma\left(\varphi^{\prime} \rightarrow \ell^{+} \ell^{-}\right)=\frac{\sin ^{2} \theta G_{F}}{\sqrt{2}} \frac{m_{\varphi^{\prime}} m_{\ell}^{2}}{4 \pi}\left(1-4 r_{\ell}^{\prime}\right)^{3 / 2}
$$

where $r_{\ell}^{\prime}=m_{\ell}^{2} / m_{\phi^{\prime}}^{2}$

\section{THREE-PORTAL PHENOMENOLOGY}

We now present the most relevant signatures arising from the interplay between portal couplings and the heavy neutrinos $\nu_{h}(h=4,5)$. We begin with a discussion of a light dark photon scenario, where we present a novel search for exotic meson decays that can be performed at NA62. Later, we comment on how the usual searches for each particle change with respect to the minimal models, separating our discussion into heavy neutrino, dark photon, and dark scalar searches. For each particle, we then point out how their modified behavior in a three-portal model would fit into recently proposed explanations to outstanding experimental anomalies.

\section{A. Exotic meson decays}

From the discussion above, it is clear that if the dark bosons are lighter than the heavy neutrinos, then $\nu_{h}$ decays are fast, and become visible whenever the decays of the light bosons are fast and visible. Because the light scalars typically decay with much longer lifetimes than the dark photons, we will always assume that they lie at much larger masses and do not play a role in our study, unless explicitly stated otherwise. As a proof of principle, we focus on a scenario with a single pseudo-Dirac heavy neutrino $\nu_{h}$ in an ISS-like regime $\left(\mu^{\prime} \rightarrow 0\right)$, although it should be noted that in this case neutrino masses vanish. The case of Majorana neutrinos that contribute to light neutrino masses is completely analogous, noting that the mass mechanism is mostly insensitive to small kinetic and scalar mixing parameters. For a comparison with the relevant region for neutrino masses, see Ref. [54].

The most striking signatures associated with the light dark photon scenario arise in kaon factories, where meson decays to heavy neutrinos lead to visible signatures inside

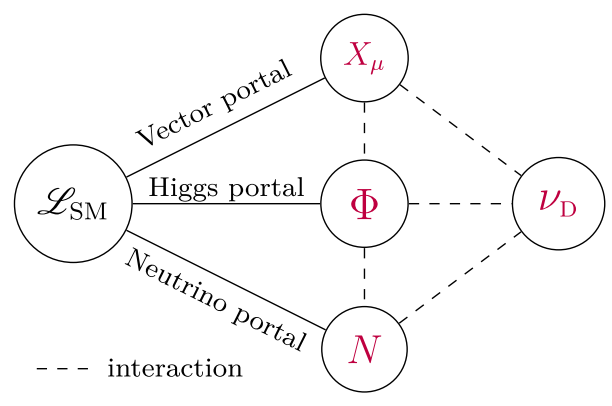

FIG. 1. Schematic representation of our three-portal model.

the detector. In this context, we propose a dedicated search for the following process:

$$
M^{+} \rightarrow \ell_{\alpha}^{+} \nu_{h} \rightarrow \ell_{\alpha}^{+} \nu Z^{\prime} \rightarrow \ell_{\alpha}^{+} \nu \ell_{\beta}^{+} \ell_{\beta}^{-},
$$

with $\alpha, \beta \in\{e, \mu\}$ (see Fig. 2). For prompt dark cascades, the $\mathrm{BR}$ for such decays is simply $\operatorname{BR}\left(M^{+} \rightarrow \ell_{\alpha}^{+} \nu_{h}\right)=$ $\left|U_{\alpha h}\right|^{2} \rho_{\alpha}\left(m_{\nu_{h}}\right) \operatorname{BR}\left(M^{+} \rightarrow \ell_{\alpha}^{+} \nu_{\alpha}\right)$, where $\rho_{\alpha}\left(m_{\nu_{h}}\right)$ is the Shrock function [75], which accounts for the heavy neutrino mass in such meson decays. This signature can be searched for by applying a simultaneous requirement of

$$
\begin{gathered}
m_{\beta \beta}^{2} \equiv\left(p_{\beta^{+}}+p_{\beta^{-}}\right)^{2} \stackrel{!}{=} m_{Z^{\prime}}^{2}, \\
m_{p_{K}-p_{\alpha}}^{2} \equiv\left(p_{K}-p_{\alpha}\right)^{2} \stackrel{!}{=} m_{\nu_{h}}^{2}, \\
m_{\mathrm{miss}}^{2} \equiv\left(p_{K}-p_{\alpha}-p_{\beta^{+}}-p_{\beta^{-}}\right)^{2} \stackrel{!}{=} 0,
\end{gathered}
$$

within detector resolution. In addition, the strong correlation and smooth distribution over the invariant masses $m_{\alpha \nu}^{2} \equiv\left(p_{K}-p_{\beta^{+}}-p_{\beta^{-}}\right)^{2}$ and $m_{\alpha \beta \beta}^{2} \equiv\left(p_{\alpha}+p_{\beta^{+}}+p_{\beta^{-}}\right)^{2}$ can be used to further reduce backgrounds. From kinematics alone, it is possible to show that

$m_{\alpha \beta \beta}^{2}+m_{\alpha \nu}^{2}+m_{p_{K}-p_{\alpha}}^{2}=m_{K}^{2}+m_{\alpha}^{2}+m_{\mathrm{miss}}^{2}+m_{\beta \beta}^{2}$,

so that a measurement of one can be tested against the other for each point in parameter space.

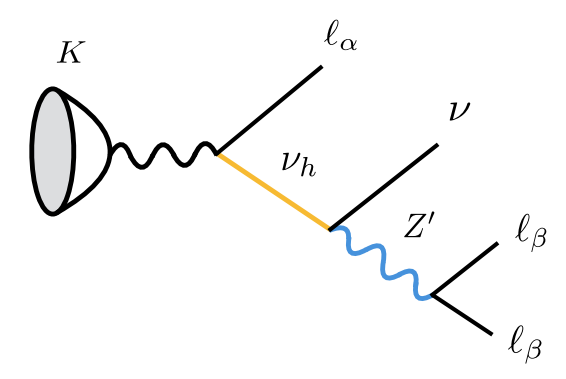

FIG. 2. Kaon decay to a heavy neutrino that decays visibly either through a sequence of two-body decays or via a threebody decay. 
Ultimately, the degree of background reduction is highly detector dependent as it arises mainly from resolution and particle identification effects. We return to this issue below, but a detailed analysis is left to the sophisticated detector simulations of the experimental collaborations. We do remark, however, that if the heavy neutral leptons (HNLs) and the dark photon have lifetimes above 10 ps in the rest frame, then they may lead to displaced vertices at NA62, as the boost factors are $\mathcal{O}(100)$ and the resolution for displaced vertices is of $\mathcal{O}(10) \mathrm{cm}$ [76]. This provides yet another tool to reduce backgrounds and is particularly useful in the small mixing region where the new physics events are expected to be small. Under the assumption of a light dark photon, a pseudo-Dirac pair that mixes only with the muon flavor, and neglecting final-state masses, the typical lifetimes are

$$
\begin{aligned}
\tau_{\nu_{h}}^{0} \simeq 1.7 \mathrm{ps} \times\left(\frac{10^{-10}}{\left|U_{D h}\right|^{2}\left|U_{\mu h}\right|^{2}}\right)\left(\frac{300 \mathrm{MeV}}{m_{\nu_{h}}}\right)^{3} \\
\times\left(\frac{0.375}{g^{\prime}}\right)^{2}\left(\frac{m_{Z^{\prime}}}{100 \mathrm{MeV}}\right)^{2} \\
\tau_{Z^{\prime}}^{0} \simeq 0.70 \mathrm{ps} \times\left(\frac{10^{-4}}{\chi}\right)^{2}\left(\frac{50 \mathrm{MeV}}{m_{Z^{\prime}}}\right),
\end{aligned}
$$

where we assumed $\operatorname{BR}\left(\nu_{h} \rightarrow \nu Z^{\prime}\right) \simeq 1$ and $\operatorname{BR}\left(Z^{\prime} \rightarrow\right.$ $\left.e^{+} e^{-}\right) \simeq 1$, with the understanding that $\left(\left|U_{D 4}\right|^{2}+\right.$ $\left.\left|U_{D 5}\right|^{2}\right)\left(1-\left|U_{D 4}\right|^{2}-\left|U_{D 5}\right|^{2}\right)=\left|U_{D h}\right|^{2}\left|U_{\mu h}\right|^{2}$ as $\mu^{\prime} \rightarrow 0$.

In what follows, we obtain an estimate of the single event sensitivity of NA62, assuming a zero-background search. We do this exercise for only $30 \%$ of the collected data set corresponding to the period between 2016 and 2018 [77]. Using $N_{K}$ useful kaon decays, downscaled by a trigger factor to yield $N_{K}^{\mathrm{Fid}}$ fiducial kaon decays, and a detector acceptance $\mathrm{A}_{\beta}$, we can find the experimental single event sensitivity to muon-heavy neutrino mixing in $K^{+} \rightarrow$ $\mu^{+} \nu \ell_{\beta} \ell_{\beta}$ decays as

$$
\left|U_{\mu h}\right|^{2}=\frac{1}{N_{K}^{\mathrm{Fid}} \mathrm{A}_{\beta} \mathrm{BR}\left(K \rightarrow \mu \nu_{\mu}\right)} \frac{1}{P_{\mathrm{dec}}},
$$

where

$$
\begin{aligned}
P_{\mathrm{dec}} \simeq & \left(1-e^{\frac{-\langle L\rangle}{L_{\nu}}}\right) \mathrm{BR}\left(\nu_{h} \rightarrow \nu Z^{\prime}\right) \\
& \times\left(1-e^{\frac{-\langle L\rangle}{L} Z^{\prime}}\right) \mathrm{BR}\left(Z^{\prime} \rightarrow \ell_{\beta} \ell_{\beta}\right)
\end{aligned}
$$

is a crude approximation for the probability for $\nu_{h}$ and $Z^{\prime}$ to decay within the average distance between their production and the end of the detector, $\langle L\rangle$, when they have a decay length $L_{P}$ in the laboratory frame. For concreteness, we take $\langle L\rangle=37.5 \mathrm{~m}$. At NA62, $\beta=e$ events ought to be registered by the "dielectron" trigger and $\beta=\mu$ by the "dimuon" trigger [77], each collecting only a fraction of the
TABLE I. Assumptions used for the computation of NA62 single event sensitivity for heavy neutrinos decaying via twobody cascades into two charged leptons plus missing energy.

\begin{tabular}{lcc}
\hline \hline & $K^{+} \rightarrow \mu^{+} \nu e^{+} e^{-}$ & $K^{+} \rightarrow \mu^{+} \nu \mu^{+} \mu^{-}$ \\
\hline$N_{K}^{\mathrm{Fid}}$ & $2.14 \times 10^{11}$ & $7.94 \times 10^{11}$ \\
$\mathrm{~A}_{\beta}$ & $4 \%$ & $10 \%$ \\
\hline \hline
\end{tabular}

total useful kaon decays, and corresponding to a given $N_{K}^{\mathrm{Fid}}$ kaon decays in the fiducial volume, as shown in Table I. We assume a constant acceptance $\mathrm{A}_{\beta}$ for each final state that is approximately the one obtained in searches for $K^{+} \rightarrow$ $\pi^{-} \ell_{\beta}^{+} \ell_{\beta}^{+}$[77] and $K^{+} \rightarrow \pi^{+}\left(\pi^{0} \rightarrow \gamma\left(Z^{\prime} \rightarrow e^{+} e^{-}\right)\right)$[78] at NA62, also shown in Table I.

Our results are shown in Fig. 3. It is clear that within our assumption of no backgrounds, NA62 can probe a large region of unexplored parameter space. Of most relevance is the region at low $m_{Z^{\prime}}$, where other bounds are rather weak. Incidentally, the dielectron search would directly test the model proposed in Ref. [43] (see MiniBooNE discussion below), where a more phenomenological model with a single heavy neutrino was used. There, all dark couplings are fixed to be large, with $\left|U_{\mu 4}\right|^{2} \gtrsim 10^{-9}$ and $m_{4} \gtrsim 30 \mathrm{MeV}$. Fixing $g^{\prime}$ to be $\mathcal{O}(1)$ implies that heavy neutrinos decay even faster, and the sensitivity curve in Fig. 3 would apply as shown. In our figure, however, we choose to fix $v_{\varphi}$ so that $g^{\prime}$ must vary if $m_{Z^{\prime}}$ varies. If one were to fix $g^{\prime}$, only the upscattering cross section region in the plot would be affected, as the cross sections are proportional to $\left(g^{\prime} e \chi\right)^{2}\left|U_{\mu h}^{*} U_{D h}\right|^{2}$.

We do not study this case here, but $K^{+} \rightarrow e^{+} \nu \ell_{\beta}^{+} \ell_{\alpha}^{-}$can also be searched for, and would lead to bounds on the $\left|U_{e h}\right|^{2}$ mixing angle, which for promptly decaying Dirac neutrinos is also weakly constrained. Much heavier $Z^{\prime}$ particles imply that heavy neutrino decay is a three-body process, and may not be so prompt. In that case, the resonance in $m_{\beta \beta}$ is no longer present, making background reduction more challenging. A peak search in $m_{p_{K}-p_{\alpha}}^{2}$ can still be performed, together with the requirement that the invariant masses measured obey Eq. (21), which is still valid. The lifetimes of $\nu_{h}$ in the ISS-like regime of our model are too long to realize this signature, but in an ESSlike regime, $\nu_{5} \rightarrow \nu_{4} \ell_{\beta}^{+} \ell_{\beta}^{-}$decays are sufficiently fast.

\section{Backgrounds}

We now discuss backgrounds to our proposed search. An irreducible but smooth background from SM radiative decays $M^{+} \rightarrow \ell_{\alpha}^{+} \nu \ell_{\beta}^{+} \ell_{\beta}^{-}$exists at a $\mathrm{BR}$ of $\mathcal{O}\left(10^{-8}\right)$, also displaying $m_{\text {miss }}^{2}=0$. Our signal, however, constitutes a single peak in the $m_{\beta \beta}$ vs $m_{p_{K}-p_{\alpha}}$ plane. Given that NA62 has already achieved an invariant mass resolution of $\delta m_{p_{K}-p_{\alpha}}=1-20 \mathrm{MeV}$ [81], depending on $m_{p_{K}-p_{\alpha}}$, and 

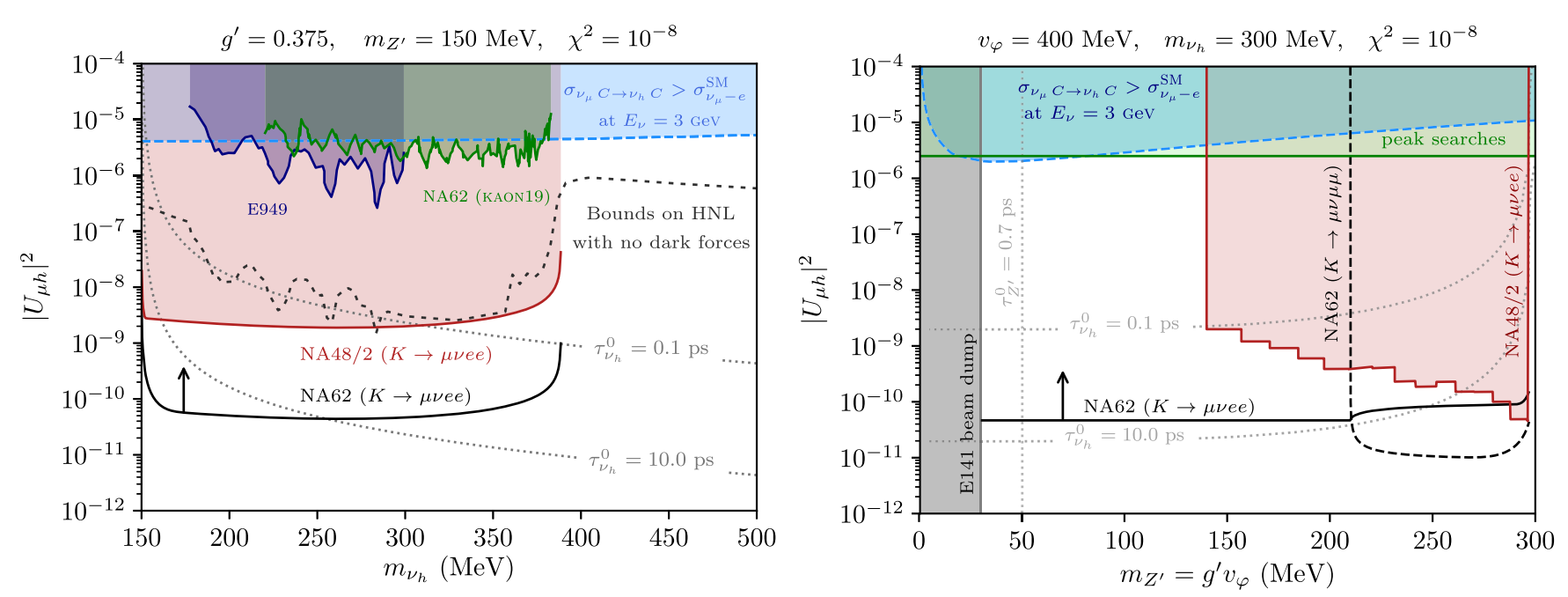

FIG. 3. Parameter space in a phenomenological ISS scenario $\left(\mu^{\prime} \rightarrow 0\right)$ where a pseudo-Dirac pair decays visibly via $\nu_{h} \rightarrow \nu Z^{\prime} \rightarrow \nu e^{+} e^{-}$. On the left we fix $m_{Z^{\prime}}$ and vary $m_{\nu_{h}}$, while on the right we fix $m_{\nu_{h}}$ and vary $m_{Z^{\prime}}$. The NA62 single event sensitivities to $K^{+} \rightarrow \mu^{+} e^{+} e^{-} \nu$ and $K^{+} \rightarrow \mu^{+} \mu^{+} \mu^{-} \nu$ are shown as solid and dashed black lines, respectively. The resonances in $m_{\ell \ell}=m_{Z^{\prime}}$ and $m_{p_{K}-p_{\mu}}=m_{\nu_{h}}$ are expected to greatly reduce backgrounds, although the region $m_{e e}<140 \mathrm{MeV}$ can still be challenging due to the large number of $\pi^{0}$ Dalitz decays. We show bounds obtained from the NA48/2 measurement of the rare leptonic kaon decay $K^{+} \rightarrow \mu \nu e^{+} e^{-}$in red [79]. Peak-search constraints on $\nu_{h}$ become less effective due to the fast visible decays, and we rescale these assuming a conservative $0.5 \% e^{+} e^{-}$detection inefficiency. We show the region where the upscattering cross section is larger than that of neutrino-electron scattering in light blue, implying that $e^{+} e^{-}$pairs could be searched for in accelerator neutrino scattering experiments, such as MINER $\nu \mathrm{A}$ [44]. Beam dump constraints on $\nu_{h}$ disappear, but those on a light $Z^{\prime}$ remain (for $\chi^{2}=10^{-8}$, E141 [80] provides the strongest bound). The dotted grey contours show a fixed total lifetime for $\nu_{h}$ and $Z^{\prime}$. For $\left|U_{\mu h}\right|>10^{-4}$, invisible $Z^{\prime} \rightarrow \nu \bar{\nu}$ decays start to dominate.

that NA48/2 has achieved $\delta m_{e e} / m_{e e} \simeq 1 \%$ [82], we expect radiative leptonic kaon decays to not limit the sensitivity once Eq. (21) has been taken into account. For $K^{+} \rightarrow$ $\mu^{+} \nu e^{+} e^{-}$, the most challenging background appears at $m_{e e}<m_{\pi^{0}}$, where the large number of $K^{+} \rightarrow \mu^{+} \nu_{\mu}\left(\pi^{0} \rightarrow\right.$ $\gamma e^{+} e^{-}$) decays can spoof our signature due to the soft nature of the photon in the pion Dalitz decay. The BR of such decays to fake our final states can be naively estimated to be around $10^{-8}$ for the excellent photon rejection at NA62 of $\simeq 10^{-4}$. This, again, can be further reduced by enforcing invariant mass peaks and correlations. Similar considerations apply for any channel with Dalitz decays. Other backgrounds from $K^{+} \rightarrow\left(\pi^{+} \rightarrow \mu^{+} \nu\right) e^{+} e^{-}$, for instance, may be reduced with a cut on $m_{\mu \nu}>m_{\pi^{+}}$, at the cost of reducing signal acceptance. While our assumption of no backgrounds is optimistic at low $m_{Z^{\prime}}$ values, the region with $m_{Z^{\prime}}>140 \mathrm{MeV}$ has been studied before in the context of rare leptonic kaon decays, and presents more manageable backgrounds. For instance, the NA48/2 Collaboration [79] has performed a measurement of

$\mathrm{BR}\left(K^{+} \rightarrow \mu^{+} \nu e^{+} e^{-}\right)=(7.81 \pm 0.21$ stat. $) \times 10^{-8}$

for $m_{e e}>140 \mathrm{MeV}$, where we omitted the smaller systematic uncertainties. This measurement achieved an overall signal-to-background ratio of $\simeq 30$, with an estimate of 54 backgrounds events.
For the dimuon channel, $K^{+} \rightarrow \pi^{+} \pi^{+} \pi^{-}$presents the largest background rate, followed by $K^{+} \rightarrow \mu^{+} \nu \pi^{+} \pi^{-}$. These channels are challenging due to the subsequent decays of the $\pi^{ \pm} \rightarrow \mu^{ \pm} \nu$ as well as the misidentification rate of $\pi \rightleftharpoons \mu$ of $0.4-0.9 \%$ at NA62. The radiative leptonic decay $K^{+} \rightarrow \mu^{+} \nu \mu^{+} \mu^{-}$would also present a background. No measurement of such SM decays exists but the most stringent limit comes from E787, where it was found that

$$
\mathrm{BR}\left(K^{+} \rightarrow \mu^{+} \nu \mu^{+} \mu^{-}\right)<4.7 \times 10^{-7}
$$

at $90 \%$ C.L. for $m_{\mu \mu} \in[220,320] \mathrm{MeV}$ [83]. NA62 will measure such SM decays, as shown in Ref. [84] which explored placing constraints on new light $L_{\mu}-L_{\tau}$ mediators. In comparison with the latter study, the multiple invariant mass resonances in our model would further reduce backgrounds and offer valuable insight if the light dark photon can decay to muons. A simultaneous detection in the electron and muon channels in accordance with the dark photon BRs would offer strong evidence for our kind of dark sector.

\section{Similar measurements}

A bound can already be derived from existing measurements compatible with the SM prediction in kaon radiative leptonic decays $[79,85,86]$. The NA48/2 Collaboration has measured the BR of $K^{+} \rightarrow \mu^{+} \nu e^{+} e^{-}$as a function of $m_{e e}$, 
starting from $m_{e e}>140 \mathrm{MeV}$, and great agreement between Monte Carlo simulations and data is observed [79]. This allows us to set constraints on $m_{Z^{\prime}}>140 \mathrm{MeV}$, for various values of $m_{\nu_{h}}$. We show the region where the new physics events are larger than $20 \%$ of the measured value for $m_{Z^{\prime}}=150 \mathrm{MeV}$ in the left panel of Fig. 3. The same is done for $m_{\nu_{h}}=300 \mathrm{MeV}$ and varying $m_{Z^{\prime}}$ in the right panel. Note that for $2 m_{\mu}<m_{Z^{\prime}}$, one would predict $K^{+} \rightarrow \mu^{+} \nu \mu^{+} \mu^{-}$decay, which results in a rather weak

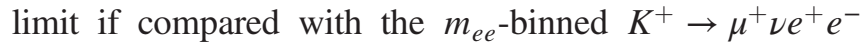
measurement at NA48/2.

Finally, similar signatures can arise in pion decays $\pi^{+} \rightarrow \ell_{\alpha}^{+} \nu e^{+} e^{-}$. For $\alpha=e$, a measurement compatible with the SM prediction was performed at the SINDRUM magnetic spectrometer [87] where

$$
\mathrm{BR}\left(\pi^{+} \rightarrow e^{+} \nu e^{+} e^{-}\right)=(3.4 \pm 0.5) \times 10^{-9} .
$$

No measurement or limit exists for $\pi^{+} \rightarrow \mu^{+} \nu e^{+} e^{-}$, where only $m_{\nu_{h}} \lesssim 34 \mathrm{MeV}$ heavy neutrinos can be tested. Productions in muon [88] and tau [89] leptonic decays of the type $\ell_{\alpha} \rightarrow \ell_{\beta} \ell_{\gamma} \ell_{\gamma} \nu \bar{\nu}$ with BRs of $\mathcal{O}\left(10^{-5}\right)$ are much less constraining, although tau decays would offer a unique process to test the less constrained $\left|U_{\tau h}\right|$ parameter.

\section{B. Impact on heavy neutrino searches}

In this subsection and the ones that follow it, we will discuss a collection of interesting signatures and the most relevant changes to the current experimental landscape in a model such ours, where at least two portal couplings may be at play. We do not make the assumption of a pseudoDirac HNL anymore, and use $\nu_{h}$ to denote both $\nu_{4}$ and $\nu_{5}$, whenever the distinction is not important. In the presence of only neutrino mixing, the strongest bounds on heavy neutrinos in the $\mathrm{MeV}-\mathrm{GeV}$ mass range come from peak searches in meson decays [90-92] and beam dump experiments [93-98] looking for visible $\nu_{h}$ decays. As we will see, both searches can be weakened if the $\nu_{h}$ decays are sufficiently different from the case of "standard" sterile neutrinos with SM interactions suppressed by neutrino mixing.

\section{Peak searches and $L N V$}

Peak searches in meson decays of the type $M \rightarrow \ell \nu_{h}$ have long been regarded as model-independent bounds on heavy neutrinos. This is due to the fact that only the parent meson and daughter charged lepton kinematics need to be measured in order to search for a peak in $\left(p_{M}-p_{\ell}\right)^{2}=m_{\nu_{h}}^{2}$. We argue that the strict requirement of observing a single charged track in the detector [91] would, however, veto a large fraction of new physics events if $\nu_{h}$ decays promptly into $\nu_{\alpha} \ell^{+} \ell^{-}$, for instance. This is not a concern in minimal sterile neutrino extensions of the SM due to the large lifetime of $\nu_{h}$, which is greatly reduced for large values of $\chi$ in our model. We illustrate this effect in the left panel of Fig. 3, where peak search bounds on $\left|U_{\mu h}\right|^{2}$ derived from $K^{+} \rightarrow \mu^{+} \nu_{h}$ decays at KEK [90], E949 [91], and NA62 $[81,99]$ are reinterpreted in an ISS-like BP where a single heavy neutrino of Dirac nature undergoes twobody decays into $\nu e^{+} e^{-}$final states. With a probability to miss an $e^{+} e^{-}$pair in the preceding detectors of $0.5 \%$, this represents a 200 times weaker bound on the mixing angle. This inefficiency is clearly a conservative assumption based on the photon detection inefficiency (typically larger than that of an $e^{+} e^{-}$pair of the same energy) reported by the E949 [91] and NA62 [100] collaborations. In addition, bounds on lepton number violation (LNV) from meson and tau decays are affected $[74,101]$. These bounds are obtained by searching for $M^{+} \rightarrow \ell^{+} \nu_{h} \rightarrow \ell^{+} \ell^{+} \pi^{-}$decays, with the same-sign dileptons being a smoking gun for LNV by two units. In our current model, the intermediate on-shell $\nu_{h}$ produced in said decays has very suppressed chargedcurrent branching ratios into $\ell^{ \pm} \pi^{\mp}$ and $\ell^{ \pm} K^{\mp}$ final states, and so such bounds are much weaker.

\section{Beam dump searches}

Beam dump and fixed target experiments are an ideal place to search for heavy neutrinos due to the large number of meson decays involved. The search strategy is based on producing such particles in decays at the target, and searching for their visible decay products in a detector located at a fixed distance from the target. If heavy neutrinos decay into visible particles faster than in the minimal sterile neutrino model, then such constraints on the mixing angle of heavy neutrinos are affected. As shown in Ref. [102], if one only enhances the decay $\Gamma\left(\nu_{h} \rightarrow\right.$ vee $)$ by a factor $\alpha$ with respect to its value in the minimal sterile model, then the upper bounds become stronger by a factor $\simeq \sqrt{\alpha}$, while the lower bounds become weaker by a factor $\simeq \alpha$. This can be understood by noting that in the vicinity of the upper bound the number of events is proportional to $\left|U_{\alpha h}\right|^{4}$, while near the lower bound it is proportional to $\left|U_{\alpha h}\right|^{2}$, as most particles decay inside the detector. For very short decay lengths, no bound can be placed, as all particles decay before reaching the detector. For this reason, beam dump bounds on $\nu_{5}$ typically vanish, while those on $\nu_{4}$ become stronger in an ESS-like regime. This typically introduces problems with searches at PS-191 [93], but can be evaded in nonminimal realizations of our model where the correlation between the mixing parameters of $\nu_{4}$ and $\nu_{5}$ is broken. We come back to this issue when discussing MiniBooNE.

\section{Exotic neutrino scattering signatures}

The presence of a light vector mediator and kinetic mixing can also induce new neutrino scattering signatures. For a hadronic target $\mathcal{H}$, light neutrinos may upscatter electromagnetically via the $Z^{\prime}$ into $\nu_{h}$, which subsequently 
decays into observable particles, e.g., $\nu_{\alpha} \mathcal{H} \rightarrow\left(\nu_{h} \rightarrow\right.$ $\left.\nu \ell_{\beta}^{+} \ell_{\beta}^{-}\right) \mathcal{H}$. Beyond offering a novel scenario to explain the MiniBooNE low-energy excess (see below), such upscattering can also produce exotic final states in neutrino detectors such as $e^{+} e^{-}, \mu^{+} \mu^{-}$, multimeson, and $\tau^{+} \tau^{-}$final states. If $m_{Z^{\prime}}<m_{\nu_{h}}$, the on-shell $Z^{\prime}$ decays can be searched for by looking for neutrino-produced dileptons with $m_{\ell \ell}=$ $m_{Z^{\prime}}$. Dimuon production, however, is tightly constrained due to kaon decay bounds (see above), unless $m_{\nu_{h}} \gtrsim$ $400 \mathrm{MeV}$. In that case, signatures from $Z^{\prime} \rightarrow \pi^{+} \pi^{-}$decays would appear for $2 m_{\pi^{+}}<m_{Z^{\prime}} \mathrm{MeV}$. If $m_{Z^{\prime}} \lesssim 140 \mathrm{MeV}$, neutrino upscattering on nuclei is predominantly coherent, and leads exclusively to $e^{+} e^{-}$final states. These can mimic single-photon showers in neutrino detectors, and could affect sideband measurements in neutrino-electron scattering data at (multi-)GeV energies (see Ref. [44]). Assuming an ISS-like model and a light dark photon, we show the region where the coherent upscattering cross section on carbon $\left(\nu_{\mu} C \rightarrow \nu_{h} C\right)$ is larger than that of neutrino-electron scattering in the SM in Fig. 3. When $m_{Z^{\prime}} \gtrsim 800 \mathrm{MeV}$, upscattering happens predominantly on protons. In that case, more hadronic activity in the detector is expected, and neutrino-electron scattering measurements are no longer as effective. If $2 m_{\mu}<m_{Z^{\prime}}<m_{\nu_{h}}$ or $2 m_{\mu}<m_{\nu_{h}}<m_{Z^{\prime}}$, then such events contribute to neutrino-trident production $\left(\nu_{\mu} \mathcal{H} \rightarrow \nu_{\mu} \mu^{+} \mu^{-} \mathcal{H}\right)$ at neutrino scattering experiments if $\nu_{h}$ decays fast enough. Previous measurements at CCFR [103] and CHARM-II [104] led to weak bounds in the parameter space shown in Fig. 3, so we do not show them here. They become relevant when $m_{Z^{\prime}} \gtrsim 800 \mathrm{MeV}$ as neutrino-electron scattering becomes ineffective.

\section{MiniBooNE low-energy excess}

The upscattering signatures discussed above with $\ell^{ \pm}=$ $e^{ \pm}$have been invoked as an explanation of the excess of low-energy electron-like events at MiniBooNE [35] in Refs. [43] and [42], where a simplified model containing a single heavy neutrino was used. This explanation relies on the fact that collimated or highly energy-asymmetric $e^{+} e^{-}$ pairs can mimic an electron-like signature in Cherenkov detectors, such as MiniBooNE. While the light dark photon case $\left(m_{Z^{\prime}}<m_{\nu_{4}}\right)$ [43] leads to tension with either the angular distribution at MiniBooNE or $\nu-e$ scattering data [44], the heavy dark photon study $\left(m_{\nu_{4}}<m_{Z^{\prime}}\right)$ [42] finds a good fit to both energy and angular distributions at MiniBooNE, while evading constraints from $\nu-e$ scattering data. The preferred parameters were $m_{4}=140 \mathrm{MeV}$, $m_{Z^{\prime}}=1.25 \mathrm{GeV}, g^{\prime}=1,\left|U_{\mu 4}\right|^{2}=1.5 \times 10^{-6}$, and $\chi^{2}=$ $5 \times 10^{-6}$. There, the prompt decays of $\nu_{4} \rightarrow \nu e^{+} e^{-}$were achieved by requiring a large mixing with the tau flavor, namely, $\left|U_{\tau 4}\right|^{2}=7.8 \times 10^{-4}$. We note, however, that the decay lengths achieved by Ref. [42] are too long when computed using our expressions, and may indicate that much larger values of dark couplings were used than the ones quoted. In addition, choosing values of $\left|U_{\tau 4}\right|^{2}=$ $520 \times\left|U_{\mu 4}\right|^{2}$ or larger would imply that experiments with a large component of $\nu_{\tau}$ neutrinos in their beam would see $\nu_{4}$ production at a comparable rate to their total neutralcurrent elastic scattering rate. With a similar $e^{+} e^{-}$-electron misidentification invoked to explain the MiniBooNE excess, such events would lead to large numbers of $\nu_{e^{-}}$ like events at the far detector of $\mathrm{T} 2 \mathrm{~K}$, in contradiction with $\nu_{e}$ appearance measurements ${ }^{1}$ [105].

In an ESS-like limit of our current model, we predict that both $\nu_{4}$ and $\nu_{5}$ can be produced in upscattering. Due to the larger $\nu_{5}-\nu_{4}-Z^{\prime}$ coupling compared to that of $\nu_{4}-$ $\nu_{i}-Z^{\prime}$, we can do away with the need for a large $\left|U_{\tau h}\right|^{2}$ mixing by producing sufficient numbers of $\nu_{5}$ states in the MiniBooNE detector. We take an analogous benchmark point to that of Ref. [42]:

$$
\begin{gathered}
m_{4}=80 \mathrm{MeV}, \quad m_{5}=140 \mathrm{MeV}, \\
\left|U_{\mu 5}\right|^{2}=4 / 7 \times\left|U_{\mu 4}\right|^{2}=1.5 \times 10^{-6}, \\
\left|U_{D 4}\right|^{2}=7 / 4 \times\left|U_{D 5}\right|^{2}=7 / 11, \\
g^{\prime}=2, \quad \chi^{2}=1 \times 10^{-5}, \quad \text { and } \quad m_{Z^{\prime}}=1.25 \mathrm{GeV} .
\end{gathered}
$$

For these parameters, we find that $\nu_{4}$ is very long-lived $\left(c \tau_{4}^{0} \simeq 30 \mathrm{~km}\right)$ and its production only introduces a small deviation from the total number of neutral-current events due to $\nu_{\mu} \mathcal{H} \rightarrow \nu_{4} \mathcal{H}$. On the other hand, the signature

$$
\nu_{\mu} \mathcal{H} \rightarrow\left(\nu_{5} \rightarrow \nu_{4} e^{+} e^{-}\right) \mathcal{H}
$$

fakes the MiniBooNE signal due to prompt $\nu_{5}$ decays $\left(c \tau_{5}^{0} \simeq 76 \mathrm{~cm}\right)$. Decays of the type $\nu_{5} \rightarrow \nu_{4} \nu \nu$ are doubly suppressed by the small mixing angles, ${ }^{2}$ and have the second largest $\mathrm{BR}$ of $\simeq 8 \times 10^{-5}$. The angular spectrum is analogous to weakly scattering neutrinos due to the large value of $m_{Z^{\prime}}=1.25 \mathrm{GeV}$, but contains only a vectorial piece coupling to matter. We also expect a larger efficiency to select signal events in our model, as we predict a larger number of events with overlapping showers (defined approximately as $\Delta \theta_{e e} \lesssim 13^{\circ}$ at MiniBooNE [35], where $\Delta \theta_{e e}$ is the opening angle of the two electrons) as well as events that are highly asymmetric in energy, both being categories that fake single electromagnetic shower events. This is due to the low invariant masses $m_{e e}$ that are now bounded by $m_{e e}<m_{5}-m_{4}=60 \mathrm{MeV}$, compared with $m_{e e}<m_{4}=140 \mathrm{MeV}$ in Ref. [42]. For the parameters

\footnotetext{
${ }^{1}$ We thank Pedro Machado for bringing this argument to our attention.

${ }^{2}$ In fact, even if the intermediate state becomes lighter, say, $m_{4}=50 \mathrm{MeV}$, the $\nu_{5} \rightarrow \nu_{4} \nu_{4} \nu \mathrm{BR}$ would be comparable to that of $\nu_{5} \rightarrow \nu_{4} e^{+} e^{-}$. Decays of the type $\nu_{5} \rightarrow \nu_{4} \nu_{4} \nu_{(4)}$ dominate whenever $3 m_{4}<m_{5}$.
} 
quoted above, we predict a total number of upscattering events on carbon plus protons in the full MiniBooNE detector of $\simeq 6.3 \times 10^{3}$ for $\nu_{4}$, and $\simeq 6.1 \times 10^{3}$ for $\nu_{5}$, before detection and signal efficiencies. These numbers correspond to $12.84 \times 10^{20}$ protons on target in neutrino mode for $818 \mathrm{t}$ of $\mathrm{CH}$, and overall efficiencies are expected to be of the order of $5 \%$. We note that our rate differs from that of Ref. [42], and that the source of discrepancy is still unknown but likely to be due to larger dark couplings than the ones quoted by the authors. The upscattering rate via $Z^{\prime}$ exchange is proportional to $\left|\sum_{i}^{3} U_{\mu i}^{*} U_{D i}\right|^{2}=$ $\left|U_{\mu 4}^{*} U_{D 4}+U_{\mu 5}^{*} U_{D 5}\right|^{2}$ and for our parameters we find negligible interference with SM bosons.

Beam dump constraints on such an unstable $\nu_{5}$ particle disappear completely and peak searches are weakened, as discussed above. For $\nu_{4}$, the only relevant constraint is that posed by the beam dump experiment PS-191 [93], where production through $K^{+} \rightarrow \mu^{+} \nu_{4}$ and decay via the $Z^{\prime}$ enhanced channel $\nu_{4} \rightarrow \nu e^{+} e^{-}$would take place. Although the $\left|U_{\mu 4}\right|^{2}$ parameter plays no role in the MiniBooNE explanation, it is heavily correlated with $\left|U_{\mu 5}\right|^{2}$ in our threeneutrino theory. Therefore, we can conclude that our benchmark point is most likely in contradiction with the constraint posed by PS-191 on $\left|U_{\mu 4}\right|^{2}$, unless such correlation is broken. A more quantitative estimate of the tension is challenging as no event selection information was provided in the original PS-191 analysis and since it assumed only charged-current decays of heavy neutrinos. We also note that an excess of electron-like events with additional tracks was later reported and attributed to neutrino interactions inside the detector [106-108] (see also the discussion in Ref. [109]).

Finally, let us emphasize that the parameters chosen in Eq. (30) are within the range of mass and mixings required to explain light neutrino masses. For instance, assuming a $2 \mathrm{GeV}$ scalar, we find that $m_{\nu} \simeq 0.4 \mathrm{eV}$ for Eq. (30), according to Eq. (8) of Ref. [54].

\section{Impact on dark photon searches}

Bounds on the vector portal come from several different sources $[110,111]$. Electroweak precision data and measurements of the $(g-2)$ of the muon and electron constrain our model [112]. Major efforts at collider and beam dump experiments led to strong constraints on dark photons by searching for the production and decay of these particles. Such bounds, however, depend on the lifetime of the $Z^{\prime}$ and on its BR into charged particles. In our model, the $Z^{\prime}$ decays invisibly into heavy fermions if $2 m_{4}<m_{Z^{\prime}}$ and into light neutrinos otherwise. In the latter case, constraints would be much weaker than usually quoted when considering only monophoton searches [113]. In the former case, however, new signatures arise, where the subsequent decay of $\nu_{h}$ leads to multilepton/multimeson events, potentially with displaced vertices and providing a very clean experimental signature. Notably, if the $Z^{\prime}$ decays into $\nu_{h}$ states that subsequently decay sufficiently fast within the detector, even the "invisible decay" bounds will be weakened.

\section{Revisiting $\Delta a_{\mu}$}

The above possibility opens the option to explain the discrepancy between the theoretical prediction [45-49] and the experimental value [50] of the $(g-2)$ of the muon via kinetic mixing. For instance, a $1 \mathrm{GeV} Z^{\prime}$ with $\chi=2.2 \times$ $10^{-2}$ can explain $a_{\mu}$. Taking $\nu_{4}$ around $800 \mathrm{MeV}$ and $m_{Z^{\prime}}<m_{5}$, the $Z^{\prime}$ would decay into $\nu_{4} \nu$ immediately. For the quoted value of the kinetic mixing and $\left|U_{\mu 4}\right|^{2}=10^{-5}$, for instance, the heavy fermions would predominantly decay with sub-cm decay lengths to $e^{+} e^{-}$and $\mu^{+} \mu^{-}$pairs plus missing energy, as well as into $\nu \rho^{0}$ around $1 \%$ of the time. This region of the $\chi$ parameter space is constrained only by the BABAR $e^{+} e^{-}$collider searches for visible [114] and invisible decays [113] of a standard dark photon. Both of these searches would veto the three-body decays of $\nu_{4}$, opening up a large region of parameter space (see Refs. [115,116] for a similar discussion in inelastic DM models). Resonance searches still constrain the $Z^{\prime}$ BR into $e^{+} e^{-}$and $\mu^{+} \mu^{-}$which are proportional to $\chi^{2}$, providing a weak upper bound. A detailed analysis to identify the viable parameter space will be done elsewhere. Note that neutrino masses are too large for the mixing quoted, but can easily be accommodated by extending the neutral fermion sector.

\section{E. Impact on dark scalar searches}

For the scalar portal, the coupling $\lambda_{\Phi H}$ is rather weakly bound by electroweak precision data and the measurement of the Higgs invisible decay at the level of $\lambda_{\Phi H} \lesssim 0.1$ [117]. For processes involving $\lambda_{\Phi H}$, the physical observables are suppressed by mass insertions due to the nature of the Higgs interaction. Nevertheless, $\varphi^{\prime}$ may decay to a pair of $\nu_{h}$ states and lead to multilepton signatures inherited from $\nu_{h}$ decays, potentially also in the form of displaced vertices. For this reason, bounds on visible decays of $\varphi^{\prime}$ no longer apply, and the parameter space $\left(m_{\varphi^{\prime}}, \sin \theta\right)$ is wide open when $\varphi^{\prime} \rightarrow \nu_{h} \nu_{h} \rightarrow$ visible happens on the cm scale.

\section{Unexplained KOTO events}

The new scalar decays can be invoked to explain the recent anomalous number of $K_{L} \rightarrow \pi^{0} \nu \bar{\nu}$ events reported by the KOTO Collaboration [53]. In our model, this can be achieved in the range $m_{\varphi^{\prime}}=120-170 \mathrm{MeV}$ and $\theta^{2} \simeq$ $4 \times 10^{-7}$, evading $K^{+} \rightarrow \pi^{+} \varphi^{\prime}$ searches at NA62 [118] and E949 [119] bounds due to large pion backgrounds at $\left(p_{K}-p_{\pi}\right)^{2} \simeq m_{\pi}^{2}$ [120]. In addition, if $\varphi^{\prime} \rightarrow \nu_{h} \nu$ decays are fast, then two scenarios arise. First, if $\nu_{4}$ is very long-lived, e.g., $m_{5} \gg m_{4}=100 \mathrm{MeV}, \chi \rightarrow 0$, and $\left|U_{\alpha}\right|^{2} \lesssim 10^{-6}$, then beam dump constraints on $\varphi^{\prime}$, such as the ones posed by the CHARM experiment [121], are relaxed. Second, if $\nu_{h}$ 
is short-lived, then one may invoke the "lifetime gap" explanation proposed in Ref. [122]. In this case, the dark scalar can decay visibly through a dark cascade with

$$
c \tau^{0}\left(\varphi^{\prime} \rightarrow \nu_{4} \nu \rightarrow \nu \nu Z^{\prime} \rightarrow \nu \nu e^{+} e^{-}\right) \simeq 10 \mathrm{~cm},
$$

and so it is vetoed in the large-acceptance NA62 and E949 detectors [118], but would inevitably count as signal for the smaller KOTO detector. The latter scenario is tightly constrained by beam dump searches at $\nu$ Cal [123-125], which, despite their large uncertainties in the region of interest [126], will be mildly alleviated due to the smaller boosts and geometrical acceptance introduced by the dark cascade. This three-portal signature with finite $\nu_{4}$ lifetime can be achieved for $g^{\prime}=0.1, \chi \simeq 2 \times 10^{-4}, m_{Z^{\prime}}=50 \mathrm{MeV}$, $m_{4}=100 \mathrm{MeV},\left|U_{\alpha 4}\right|^{2} \lesssim 10^{-10}$, and $m_{\varphi^{\prime}}=140 \mathrm{MeV}$.

\section{F. Neutrinophilic limit}

In the limiting case of a neutrinophilic model $\left(\chi=\lambda_{\Phi H}=0\right)$, the vector and scalar particles present a challenge for detection. Nonetheless, if light, they can be searched for in meson decays $[127,128]$ and at neutrino experiments [129]. Finally, the faster decays of $\nu_{h}$ and its self-interactions can help ameliorate tensions with cosmological observations. We do not comment further on this, but note that great effort has been put into accommodating eV-scale sterile neutrinos charged under new forces with cosmological observables [26-31,130] (see also Ref. [131] for an interesting discussion where the $Z^{\prime}$ decay to neutrinos leads to an altered expansion history of the Universe). We note that an $\mathrm{eV}$ sterile neutrino with relatively large mixing could be easily accommodated in our ESS framework. The $\mathrm{eV}$ neutrino would be mainly in the $\nu_{D}$ direction and would have strong hidden gauge interactions.

\section{DARK MATTER}

Given the presence of a dark sector, we can ask if the model can accommodate a DM candidate. This can be achieved by introducing new fermions that do not mix with the neutrinos, in order to preserve their stability. A minimal solution would be to introduce a fermionic field $\psi_{L}$ which has $U(1)^{\prime}$ charge $1 / 2$. The different charges of $\psi, \nu_{D}$, and $N$ would forbid neutrino mixing. A Majorana mass term

$$
\psi_{L}^{T} C^{\dagger} \psi_{L}
$$

would emerge after hidden-symmetry breaking, leading to a Majorana DM candidate. Anomaly cancellation requires additional particle content, such as promoting all charged fermions to vector-like states.

Another realization with additional symmetries can be made anomaly free. Following Ref. [65], we introduce a pair of chiral fermion fields $\psi_{L}$ and $\psi_{R}$, and charge only the latter under the $U(1)^{\prime}$ symmetry with the same charge as
$\nu_{D}$. This choice ensures anomaly cancellation, and allows us to write

$$
y_{\psi} \overline{\psi_{L}} \psi_{R} \Phi^{\dagger},
$$

which after hidden-symmetry breaking yields a Dirac mass $m_{\psi}$. In general, $\psi_{L}$ may also have a Majorana mass term $\mu_{\psi}$, giving rise to a two-component Majorana dark matter sector. Setting $\mu_{\psi} \rightarrow 0$ is technically natural, and would ensure a Dirac dark matter candidate. In order to avoid $\psi_{R}-\nu_{D}$ and $\psi_{L}-N$ mixing, an additional $\mathbb{Z}_{2}$ symmetry may be imposed, under which all particles have charge +1 , except for $\psi_{L}$ and $\psi_{R}$, which have charge -1 . This socalled dark parity can be thought of as a consequence of lepton number $L$. Since $L(\psi)=0$, the Majorana mass term for $N$ is the only soft breaking term, which breaks lepton number by $\Delta L=2$ units. Lepton number is then reduced to conservation of lepton parity $(-1)^{L}$, which can then be shown to be equivalent to our dark parity [132].

If the scalar and vector portal couplings are small in such scenarios, DM interacts mainly with neutrinos. Directdetection bounds are then evaded, since interactions with matter are loop suppressed. Indirect detection, on the other hand, is more promising as DM annihilation into neutrinos would dominate. For instance, take the mass of $\psi$ to be smaller than the masses of the $Z^{\prime}, \varphi^{\prime}$, and both heavy neutrinos. In this case, the DM annihilation is directly into light neutrinos via $\psi \bar{\psi} \rightarrow \nu_{i} \nu_{i}$. This yields a monoenergetic neutrino line that can be looked for in large-volume neutrino [133,134] or direct-detection experiments [25]. Alternatively, if $m_{\psi}$ is larger than the mass of any of our new particles, then the annihilation may be predominantly into such states via $\psi \bar{\psi} \rightarrow X X$, where $X=\varphi^{\prime}, Z^{\prime}$, or $\nu_{h}$, which subsequently decay to light neutrinos. In this secluded realization [135], the neutrino spectrum from such annihilation is continuous [61], but neutrino-DM interactions are expected to be large and can be searched for in a variety of ways [64,136-139].

\section{CONCLUSIONS}

We have proposed a new model which invokes the existence of a hidden $U(1)^{\prime}$ symmetry confined to a new dark neutrino sector. Without restricting to the region where our model generates the correct neutrino masses, we explored a series of experimental signatures that may arise from the interplay of portal couplings. The simultaneous presence of neutrino, vector kinetic, and scalar mixing in a self-consistent framework allows for a diverse phenomenology. In particular, signatures such as multilepton final states with missing energy, displaced vertices, rare leptonic decays, and unique final states in neutrino scattering processes are a hallmark of such nonminimal models. We have also argued that previously excluded parameter space for the minimal realization of each portal coupling opens up due to faster and semivisible decays in the dark 
sector. This also impacts peak searches in meson decays, often regarded as model-independent bounds. For heavy neutrinos that decay to light dark photons, we identified a unique signature at kaon experiments, wherein three charged-lepton final states can be used to search for this class of dark sectors, and in the case of a signal, reveal the heavy neutrino and dark photon masses. This search can be performed at NA62, where significant improvement over current bounds can be achieved. These searches are particularly relevant at low dilepton invariant masses $m_{e e}$, as they could further test the phenomenological model discussed in Refs. [43,44], where low-energy dielectrons produced in neutrino upscattering explain the energy distribution of the MiniBooNE excess.

The model also offers exciting new avenues to explain currently outstanding experimental anomalies. In this article, we discussed how recently proposed explanations would fit within a nonminimal dark sector like ours, each with their separate choice of parameters. For the MiniBooNE low-energy excess, we proposed a GeV-scale $Z^{\prime}$ for the angular distribution, and a pair of heavy neutrinos with $m_{4} \simeq 80 \mathrm{MeV}$ and $m_{5} \simeq 140 \mathrm{MeV}$. The decays of $\nu_{5}$ are $\mathrm{cm}$ scale, but $\nu_{4}$ travels much longer distances. Unfortunately, such $\nu_{4}$ particles have enhanced lifetimes with respect to the minimal model, and are in tension with the PS-191 beam dump experiment unless the correlation present in the three-neutrino model between the $\nu_{4}$ and $\nu_{5}$ mixing angles is broken. For heavy neutrinos with a larger mass $m_{4} \simeq 800 \mathrm{MeV}$, a $Z^{\prime}$ of $1 \mathrm{GeV}$ with $\chi=2.2 \times 10^{-2}$ can explain the discrepancy observed in the anomalous magnetic moment of the muon, $\Delta a_{\mu}$, while at the same time evading constraints from $Z^{\prime} \rightarrow$ invisible at $B A B A R$ due to the semivisible decays of $\nu_{4}$. Finally, a light scalar with $m_{\varphi^{\prime}} \simeq 140 \mathrm{MeV}$ can explain the KOTO anomaly [126]. Beam dump bounds on such scalars are completely evaded if it decays to long-lived $\nu_{4}$ states, or are mildly alleviated if it undergoes a dark cascade to $e^{+} e^{-}$within $10 \mathrm{~cm}$, the latter also evading NA62 bounds from $K^{+} \rightarrow \pi^{+} \boldsymbol{E}$ searches. A correlation between the $\left|U_{\alpha 4}\right|^{2}$ and $\left|U_{\alpha 5}\right|^{2}$ (as well as between $\left|U_{D 4}\right|^{2}$ and $\left|U_{D 5}\right|^{2}$ ) neutrino mixing parameters within our three-neutrino model precludes a simultaneous explanation of all of the aforementioned anomalies without introducing tensions with current experimental bounds. Nevertheless, the signatures we discussed pertain to a far more general class of models, and deserve further consideration due to their unique character.

\section{ACKNOWLEDGMENTS}

The authors would like to thank Martin Bauer for interesting discussions regarding the dark photon and $(g-2)_{\mu}$ phenomenology, and Andrés Olivares-del-Campo and Arsenii Titov for insights on the dark matter models. We acknowledge interesting discussions with Evgueni Goudzovski on the physics potential of NA62. This work was partially supported by Conselho Nacional de Ciência e Tecnologia $(\mathrm{CNPq})$. This project also received support from the European Union's Horizon 2020 research and innovation programme under the Marie Skłodowska-Curie Grant Agreement No. 690575 (RISE InvisiblesPlus) and No. 674896 (ITN Elusives). S. P. and P. B. are supported by the European Research Council under ERC Grant NuMass (FP7-IDEAS-ERC ERC-CG 617143). S. P. acknowledges partial support from the Wolfson Foundation and the Royal Society. The research at the Perimeter Institute is supported in part by the Government of Canada through NSERC and by the Province of Ontario through Ministry of Economic Development and Trade (MEDT).

\section{APPENDIX: KINEMATICS OF EXOTIC KAON DECAYS}

In this Appendix we expand on details of the rare kaon decay searches we propose. Beyond the peaks in $m_{\beta \beta}=$ $m_{Z^{\prime}}, m_{p_{K}-p_{\alpha}}=m_{\nu_{h}}$, and $m_{\text {miss }}=0$, one may explore the correlation and absolute range of two other relevant invariant masses, namely,

$$
\begin{gathered}
m_{\alpha \nu}^{2}=\left(p_{\mu}+p_{\nu}\right)^{2}=\left(p_{K}-p_{\beta^{+}}-p_{\beta^{-}}\right)^{2}, \\
m_{\alpha \beta \beta}^{2}=\left(p_{\alpha}+p_{\beta^{+}}+p_{\beta^{-}}\right)^{2} .
\end{gathered}
$$

Their absolute kinematical range is determined, and shown here for the reader's convenience:

$$
\begin{gathered}
\left(m_{\alpha \nu}^{2}\right)_{\min }^{\max }=m_{K}^{2}\left[1+x_{Z^{\prime}}-\frac{\left(1-x_{\alpha}+x_{h}\right)(1+y) \mp(1-y) \lambda^{1 / 2}\left(1, x_{h}, x_{\alpha}\right)}{2}\right], \\
\left(m_{\alpha \beta \beta}^{2}\right)_{\min }^{\max }=m_{K}^{2}\left[1-(1-y) \frac{\left(1-x_{\alpha}+x_{h}\right) \mp \lambda^{1 / 2}\left(1, x_{h}, x_{\alpha}\right)}{2}\right],
\end{gathered}
$$

where $\lambda(a, b, c)=(a-b-c)^{2}-4 b c$ is the Källen function, $x_{Z^{\prime}}=\left(m_{Z^{\prime}} / m_{K}\right)^{2}, x_{h}=\left(m_{\nu_{h}} / m_{K}\right)^{2}, x_{\alpha}=\left(m_{\alpha} / m_{K}\right)^{2}$, and $y=\left(m_{Z^{\prime}} / m_{\nu_{h}}\right)^{2}$.

To illustrate the properties of our signal, we develop our own toy Monte Carlo simulation, and generate $10^{4}$ events for $K^{+} \rightarrow \mu^{+}\left(\nu_{h} \rightarrow \nu\left(Z^{\prime} \rightarrow e^{+} e^{-}\right)\right)$and $K^{+} \rightarrow \mu_{1}^{+}\left(\nu_{h} \rightarrow\right.$ $\left.\nu\left(Z^{\prime} \rightarrow \mu_{2}^{+} \mu^{-}\right)\right)$decays in a few cases of interest. We fix the kaon momentum to the average value in the NA62 beam, namely, $75 \mathrm{GeV} / \mathrm{c}$. We plot the invariant masses $m_{\nu \alpha}$ and $m_{\alpha \beta \beta}$, as well as the opening angle between the leptons 

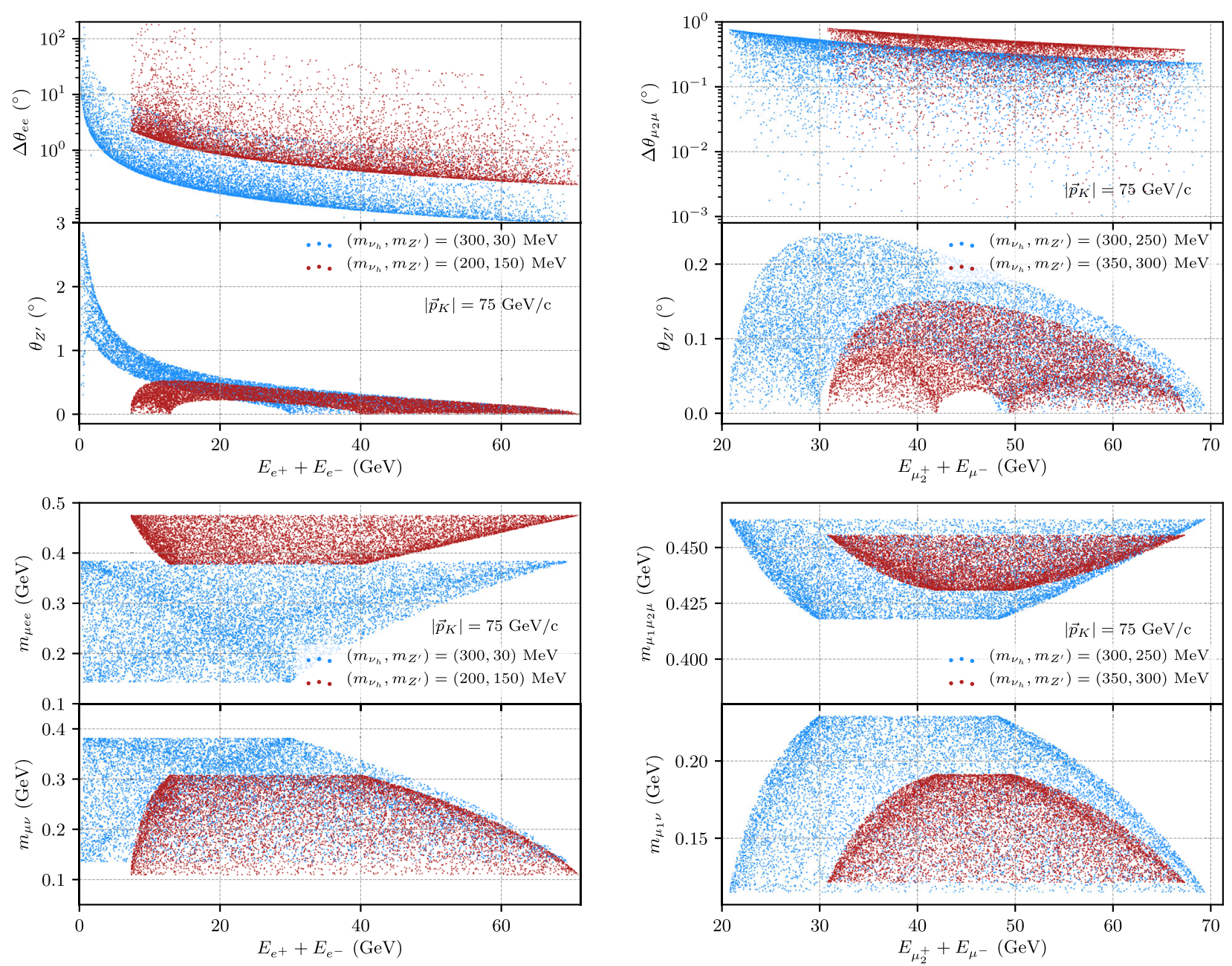

FIG. 4. Kinematics of our $K^{+} \rightarrow \mu^{+} \nu e^{+} e^{-}$(left column) and $K^{+} \rightarrow \mu_{1}^{+} \nu \mu_{2}^{+} \mu^{-}$(right column) events at kaon energies relevant to NA62. We plot $10^{4}$ events in true Monte Carlo variables in each panel. In the top row we show both the separation angle between the decay products of the $Z^{\prime}, \Delta \theta_{\beta \beta}$, and the angle of the $Z^{\prime}$ momentum with respect to the kaon beam, $\theta_{Z^{\prime}}$, both versus the total energy of the decay products of the $Z^{\prime}, E_{\beta^{+}}+E_{\beta^{-}}$. In the bottom row we show the invariant masses $m_{\mu_{(1)} \beta \beta}=\sqrt{\left(p_{\mu_{(1)}}+p_{\beta}+p_{\beta}\right)^{2}}$ and $m_{\mu_{(1)} \nu}=\sqrt{\left(p_{\left.\mu_{(1}\right)}+p_{\nu}\right)^{2}}$, also versus the total energy $E_{\beta^{+}}+E_{\beta^{-}}$.

$\Delta \theta_{\beta \beta}$, and the angle of the $Z^{\prime}$ momentum with respect to the kaon beam $\theta_{Z^{\prime}}$ versus the total $Z^{\prime}$ energy in Fig. 4. As expected, the kinematics of the signal is highly boosted, with very small angles between the decay products.
Another feature is the broad distributions in invariant masses, except for $m_{\mu_{1} \mu_{2} \mu}$, which has somewhat of a smaller range due to the limited phase space of each decay reaction.
[1] C. Boehm and P. Fayet, Nucl. Phys. B683, 219 (2004).

[2] C. Boehm, P. Fayet, and J. Silk, Phys. Rev. D 69, 101302 (2004).

[3] J. Alexander et al., arXiv:1608.08632.

[4] W. Buchmuller, C. Greub, and P. Minkowski, Phys. Lett. B 267, 395 (1991).

[5] S. Khalil, J. Phys. G 35, 055001 (2008).
[6] P. Fileviez Perez, T. Han, and T. Li, Phys. Rev. D 80, 073015 (2009).

[7] S. Khalil, Phys. Rev. D 82, 077702 (2010).

[8] C. O. Dib, G. R. Moreno, and N. A. Neill, Phys. Rev. D 90, 113003 (2014).

[9] S. Baek, H. Okada, and K. Yagyu, J. High Energy Phys. 04 (2015) 049. 
[10] V. De Romeri, E. Fernandez-Martinez, J. Gehrlein, P. A. N. Machado, and V. Niro, J. High Energy Phys. 10 (2017) 169.

[11] T. Nomura and H. Okada, Lett. High Energy Phys. 1, 10 (2018).

[12] V. Brdar and A. Y. Smirnov, J. High Energy Phys. 02 (2019) 045.

[13] H. Okada and K. Yagyu, Phys. Rev. D 90, 035019 (2014).

[14] C. E. Diaz, S. F. Mantilla, and R. Martinez, arXiv: 1712.05433 .

[15] T. Nomura and H. Okada, Phys. Rev. D 99, 055033 (2019).

[16] C. Hagedorn, J. Herrero-García, E. Molinaro, and M. A. Schmidt, J. High Energy Phys. 11 (2018) 103.

[17] B. Shakya and J. D. Wells, J. High Energy Phys. 02 (2019) 174.

[18] M. Pospelov, Phys. Rev. D 84, 085008 (2011).

[19] B. Batell, M. Pospelov, and B. Shuve, J. High Energy Phys. 08 (2016) 052.

[20] Y. Farzan, Phys. Lett. B 748, 311 (2015).

[21] Y. Farzan and J. Heeck, Phys. Rev. D 94, 053010 (2016).

[22] M. Pospelov and J. Pradler, Phys. Rev. D 85, 113016 (2012); 88, 039904(E) (2013).

[23] M. Pospelov and J. Pradler, Phys. Rev. D 89, 055012 (2014).

[24] R. Harnik, J. Kopp, and P. A. N. Machado, J. Cosmol. Astropart. Phys. 07 (2012) 026.

[25] D. McKeen and N. Raj, Phys. Rev. D 99, 103003 (2019).

[26] S. Hannestad, R. S. Hansen, and T. Tram, Phys. Rev. Lett. 112, 031802 (2014).

[27] B. Dasgupta and J. Kopp, Phys. Rev. Lett. 112, 031803 (2014).

[28] A. Mirizzi, G. Mangano, O. Pisanti, and N. Saviano, Phys. Rev. D 91, 025019 (2015).

[29] X. Chu, B. Dasgupta, and J. Kopp, J. Cosmol. Astropart. Phys. 10 (2015) 011.

[30] J.F. Cherry, A. Friedland, and I. M. Shoemaker, arXiv:1605.06506.

[31] X. Chu, B. Dasgupta, M. Dentler, J. Kopp, and N. Saviano, J. Cosmol. Astropart. Phys. 11 (2018) 049.

[32] P. B. Denton, Y. Farzan, and I. M. Shoemaker, Phys. Rev. D 99, 035003 (2019).

[33] A. Esmaili and H. Nunokawa, Eur. Phys. J. C 79, 70 (2019).

[34] A. A. Aguilar-Arevalo et al. (MiniBooNE Collaboration), Phys. Rev. Lett. 98, 231801 (2007).

[35] A. A. Aguilar-Arevalo et al. (MiniBooNE Collaboration), Phys. Rev. Lett. 121, 221801 (2018).

[36] C. Athanassopoulos et al. (LSND Collaboration), Phys. Rev. Lett. 77, 3082 (1996).

[37] A. Aguilar-Arevalo et al. (LSND Collaboration), Phys. Rev. D 64, 112007 (2001).

[38] S. N. Gninenko, Phys. Rev. Lett. 103, 241802 (2009).

[39] S. N. Gninenko, Phys. Rev. D 83, 015015 (2011).

[40] M. Masip, P. Masjuan, and D. Meloni, J. High Energy Phys. 01 (2013) 106.

[41] A. Radionov, Phys. Rev. D 88, 015016 (2013).

[42] P. Ballett, S. Pascoli, and M. Ross-Lonergan, Phys. Rev. D 99, 071701 (2019).

[43] E. Bertuzzo, S. Jana, P. A. N. Machado, and R. Zukanovich Funchal, Phys. Rev. Lett. 121, 241801 (2018).
[44] C. A. Argüelles, M. Hostert, and Y.-D. Tsai, Phys. Rev. Lett. 123, 261801 (2019).

[45] M. Davier, A. Hoecker, B. Malaescu, and Z. Zhang, Eur. Phys. J. C 71, 1515 (2011); 72, 1874(E) (2012).

[46] M. Davier, A. Hoecker, B. Malaescu, and Z. Zhang, Eur. Phys. J. C 77, 827 (2017).

[47] T. Blum, P. A. Boyle, V. Gülpers, T. Izubuchi, L. Jin, C. Jung, A. Jüttner, C. Lehner, A. Portelli, and J. T. Tsang (RBC, UKQCD Collaborations), Phys. Rev. Lett. 121, 022003 (2018).

[48] A. Keshavarzi, D. Nomura, and T. Teubner, Phys. Rev. D 97, 114025 (2018).

[49] M. Davier, A. Hoecker, B. Malaescu, and Z. Zhang, Eur. Phys. J. C 80, 241 (2020).

[50] G. W. Bennett et al. (Muon g-2 Collaboration), Phys. Rev. D 73, 072003 (2006).

[51] P. Fayet, Phys. Rev. D 75, 115017 (2007).

[52] M. Pospelov, Phys. Rev. D 80, 095002 (2009).

[53] S. Shinohara, Search for the rare decay $K_{L} \rightarrow \pi^{0} \nu \bar{\nu}$ at J-PARC KOTO experiment, in Proceedings of the kAON2019, Perugia, Italy.

[54] P. Ballett, M. Hostert, and S. Pascoli, Phys. Rev. D 99, 091701 (2019).

[55] E. Ma, Phys. Rev. D 73, 077301 (2006).

[56] Y. Farzan, Phys. Rev. D 80, 073009 (2009).

[57] Y. Farzan, S. Pascoli, and M. A. Schmidt, J. High Energy Phys. 10 (2010) 111.

[58] A. Arhrib, C. Bœhm, E. Ma, and T.-C. Yuan, J. Cosmol. Astropart. Phys. 04 (2016) 049.

[59] J. F. Cherry, A. Friedland, and I. M. Shoemaker, arXiv: 1411.1071.

[60] M. Escudero, N. Rius, and V. Sanz, J. High Energy Phys. 02 (2017) 045.

[61] M. Escudero, N. Rius, and V. Sanz, Eur. Phys. J. C 77, 397 (2017).

[62] B. Batell, T. Han, D. McKeen, and B. Shams Es Haghi, Phys. Rev. D 97, 075016 (2018).

[63] F. Capozzi, I. M. Shoemaker, and L. Vecchi, J. Cosmol. Astropart. Phys. 07 (2017) 021.

[64] A. Olivares-Del Campo, C. Bœhm, S. Palomares-Ruiz, and S. Pascoli, Phys. Rev. D 97, 075039 (2018).

[65] M. Blennow, E. Fernandez-Martinez, A. Olivares-Del Campo, S. Pascoli, S. Rosauro-Alcaraz, and A. V. Titov, Eur. Phys. J. C 79, 555 (2019).

[66] R. N. Mohapatra and J. W. F. Valle, Phys. Rev. D 34, 1642 (1986).

[67] M. C. Gonzalez-Garcia and J. W. F. Valle, Phys. Lett. B 216, 360 (1989).

[68] J. Barry, W. Rodejohann, and H. Zhang, J. High Energy Phys. 07 (2011) 091.

[69] H. Zhang, Phys. Lett. B 714, 262 (2012).

[70] P. S. B. Dev and A. Pilaftsis, Phys. Rev. D 86, 113001 (2012).

[71] V. Barger, P. Langacker, M. McCaskey, M. RamseyMusolf, and G. Shaughnessy, Phys. Rev. D 79, 015018 (2009).

[72] B. Holdom, Phys. Lett. 166B, 196 (1986).

[73] P. Ilten, Y. Soreq, M. Williams, and W. Xue, J. High Energy Phys. 06 (2018) 004.

[74] A. Atre, T. Han, S. Pascoli, and B. Zhang, J. High Energy Phys. 05 (2009) 030. 
[75] R. E. Shrock, Phys. Rev. D 24, 1232 (1981).

[76] E. Goudzovski (private communication).

[77] E. Cortina Gil et al. (NA62 Collaboration), Phys. Lett. B 797, 134794 (2019).

[78] E. Cortina Gil et al. (NA62 Collaboration), J. High Energy Phys. 05 (2019) 182.

[79] L. Peruzzo (NA48/2 Collaboration), J. Phys. Conf. Ser. 873, 012020 (2017).

[80] E. M. Riordan et al., Phys. Rev. Lett. 59, 755 (1987).

[81] E. Cortina Gil et al. (NA62 Collaboration), Phys. Lett. B 778, 137 (2018).

[82] J. Batley et al. (NA48/2 Collaboration), Phys. Lett. B 746, 178 (2015).

[83] M. S. Atiya et al., Phys. Rev. Lett. 63, 2177 (1989).

[84] G. Krnjaic, G. Marques-Tavares, D. Redigolo, and K. Tobioka, Phys. Rev. Lett. 124, 041802 (2020).

[85] A. A. Poblaguev et al., Phys. Rev. Lett. 89, 061803 (2002).

[86] H. Ma et al., Phys. Rev. D 73, 037101 (2006).

[87] S. Egli et al. (SINDRUM Collaboration), Phys. Lett. B 175, 97 (1986).

[88] W. H. Bertl et al. (SINDRUM Collaboration), Nucl. Phys. B260, 1 (1985).

[89] M. S. Alam et al. (CLEO Collaboration), Phys. Rev. Lett. 76, 2637 (1996).

[90] T. Yamazaki et al., Conf. Proc. C 840719, 262 (1984).

[91] A. V. Artamonov et al. (E949 Collaboration), Phys. Rev. D 91, 052001 (2015); 91, 059903(E) (2015).

[92] J. R. Batley et al. (NA48/2 Collaboration), Phys. Lett. B 769, 67 (2017).

[93] G. Bernardi et al., Phys. Lett. 166B, 479 (1986).

[94] F. Bergsma et al. (CHARM Collaboration), Phys. Lett. 128B, 361 (1983).

[95] J. Badier et al. (NA3 Collaboration), Z. Phys. C 31, 21 (1986).

[96] A. Vaitaitis et al. (NuTeV, E815 Collaborations), Phys. Rev. Lett. 83, 4943 (1999).

[97] A. M. Cooper-Sarkar et al. (WA66 Collaboration), Phys. Lett. 160B, 207 (1985).

[98] P. Astier et al. (NOMAD Collaboration), Phys. Lett. B 506, 27 (2001).

[99] E. Goudzovski, Exotic searches at the NA62 experiment at CERN, in Proceedings of the kAON2019, Perugia, Italy.

[100] E. Cortina Gil et al. (NA62 Collaboration), J. Instrum. 12, P05025 (2017).

[101] A. Abada, V. De Romeri, M. Lucente, A. M. Teixeira, and T. Toma, J. High Energy Phys. 02 (2018) 169.

[102] P. Ballett, S. Pascoli, and M. Ross-Lonergan, J. High Energy Phys. 04 (2017) 102.

[103] S. R. Mishra et al. (CCFR Collaboration), Phys. Rev. Lett. 66, 3117 (1991).

[104] D. Geiregat et al. (CHARM-II Collaboration), Phys. Lett. B 245, 271 (1990).

[105] K. Abe et al. (T2K Collaboration), Phys. Rev. Lett. 121, 171802 (2018).

[106] F. Vannucci (Athens-CERN-Paris-Rome Collaboration), Perspectives of Electroweak Interactions, in Proceedings of the Leptonic Session, 20th Rencontres de Moriond, Les ARCS, France, 1985, edited by J. Thanh Van Tran (Atlantica Séguier Frontières, 1985), Vol. 2, pp. 277-285.
[107] G. Bernardi et al., Phys. Lett. B 181, 173 (1986).

[108] P. Astier et al., Nucl. Phys. B335, 517 (1990).

[109] O. Fischer, A. Hernandez-Cabezudo, and T. Schwetz, Phys. Rev. D 101, 075045 (2020).

[110] D. Curtin, R. Essig, S. Gori, and J. Shelton, J. High Energy Phys. 02 (2015) 157.

[111] M. Bauer, P. Foldenauer, and J. Jaeckel, J. High Energy Phys. 07 (2018) 094.

[112] A. Hook, E. Izaguirre, and J. G. Wacker, Adv. High Energy Phys. 2011, 859762 (2011).

[113] J. P. Lees et al. (BABAR Collaboration), Phys. Rev. Lett. 119, 131804 (2017).

[114] J. P. Lees et al. (BABAR Collaboration), Phys. Rev. Lett. 113, 201801 (2014).

[115] G. Mohlabeng, Phys. Rev. D 99, 115001 (2019).

[116] M. Duerr, T. Ferber, C. Hearty, F. Kahlhoefer, K. SchmidtHoberg, and P. Tunney, J. High Energy Phys. 02 (2020) 039.

[117] A. M. Sirunyan et al. (CMS Collaboration), Phys. Lett. B 793, 520 (2019).

[118] G. Ruggiero, Latest measurement of $K^{+} \rightarrow \pi^{+} \nu \bar{\nu}$ with the NA62 experiment at CERN, in kAON2019, Perugia, Italy.

[119] A. V. Artamonov et al. (BNL-E949 Collaboration), Phys. Rev. D 79, 092004 (2009).

[120] G. W. S. Hou, J. Phys. Conf. Ser. 800, 012024 (2017).

[121] F. Bergsma et al. (CHARM Collaboration), Phys. Lett. B 157B, 458 (1985).

[122] T. Kitahara, T. Okui, G. Perez, Y. Soreq, and K. Tobioka, Phys. Rev. Lett. 124, 071801 (2020).

[123] J. Blumlein et al., Z. Phys. C 51, 341 (1991).

[124] J. Blumlein and J. Brunner, Phys. Lett. B 701, 155 (2011).

[125] J. Blümlein and J. Brunner, Phys. Lett. B 731, 320 (2014).

[126] D. Egana-Ugrinovic, S. Homiller, and P. Meade, Phys. Rev. Lett. 124, 191801 (2020).

[127] R. Laha, B. Dasgupta, and J. F. Beacom, Phys. Rev. D 89, 093025 (2014).

[128] P. Bakhti and Y. Farzan, Phys. Rev. D 95, 095008 (2017).

[129] P. Bakhti, Y. Farzan, and M. Rajaee, Phys. Rev. D 99, 055019 (2019).

[130] N. Song, M. C. Gonzalez-Garcia, and J. Salvado, J. Cosmol. Astropart. Phys. 10 (2018) 055.

[131] M. Escudero, D. Hooper, G. Krnjaic, and M. Pierre, J. High Energy Phys. 03 (2019) 071.

[132] E. Ma, Phys. Rev. Lett. 115, 011801 (2015).

[133] J. F. Beacom, N. F. Bell, and G. D. Mack, Phys. Rev. Lett. 99, 231301 (2007).

[134] S. Palomares-Ruiz and S. Pascoli, Phys. Rev. D 77, 025025 (2008).

[135] M. Pospelov, A. Ritz, and M. B. Voloshin, Phys. Lett. B 662, 53 (2008).

[136] G. Mangano, A. Melchiorri, P. Serra, A. Cooray, and M. Kamionkowski, Phys. Rev. D 74, 043517 (2006).

[137] R. J. Wilkinson, C. Boehm, and J. Lesgourgues, J. Cosmol. Astropart. Phys. 05 (2014) 011.

[138] Y. Farzan and S. Palomares-Ruiz, J. Cosmol. Astropart. Phys. 06 (2014) 014.

[139] C. A. Argüelles, A. Kheirandish, and A. C. Vincent, Phys. Rev. Lett. 119, 201801 (2017). 